\title{
A lattice Boltzmann method for axisymmetric multicomponent flows with high viscosity ratio
}

\author{
Haihu Liu ${ }^{\mathrm{a}, *}$, Lei $\mathrm{Wu}^{\mathrm{b}}$, Yan $\mathrm{Ba}^{\mathrm{a}}$, Guang $\mathrm{Xi}^{\mathrm{a}}$, Yonghao Zhang ${ }^{\mathrm{b}}$ \\ ${ }^{a}$ School of Energy and Power Engineering, Xi'an Jiaotong University, 28 West Xianning \\ Road, Xi'an 710049, China \\ ${ }^{b}$ James Weir Fluids Laboratory, Department of Mechanical $\&$ Aerospace Engineering, \\ University of Strathclyde, Glasgow G1 1XJ, UK
}

\begin{abstract}
A color-gradient lattice Boltzmann method (LBM) is proposed to simulate axisymmetric multicomponent flows. This method uses a collision operator that is a combination of three separate parts, namely single-component collision operator, perturbation operator, and recoloring operator. A source term is added into the single-component collision operator such that in each single-component region the axisymmetric continuity and momentum equations can be exactly recovered. The interfacial tension effect is realized by the perturbation operator, in which an interfacial force of axisymmetric form is derived using the concept of continuum surface force. A recoloring operator proposed by Latva-Kokko and Rothman is extended to the axisymmetric case for phase segregation and maintenance of the interface. To enhance the method's numerical stability for handling binary fluids with high viscosity ratio, a multiple-relaxation-time model is used for the collision operator. Several numerical examples, including static droplet test, oscillation of a viscous droplet, and breakup of a liquid thread, are presented to test the capability and accuracy of the proposed color-gradient LBM. It is found that the present method is able to accurately capture the phase interface and produce low spurious velocities. Also, the LBM results are all in good agreement with the analytical solutions and/or available experimental
\end{abstract}

\footnotetext{
* Corresponding author

Email address: haihu.liu@xjtu.edu.cn (Haihu Liu)
} 
data for a very broad range of viscosity ratios.

Keywords: Lattice Boltzmann method, Axisymmetric flow, Color-gradient model, High viscosity ratio, Rayleigh instability

\section{Introduction}

Multiphase, multicomponent flows of incompressible fluids are ubiquitous in nature and in many industrial processes, and have received considerable attention in the past few decades. With the development of computer hardware and advances in numerical techniques and algorithms, computational fluid dynamics (CFD) has proved to be a parctical and reliable tool for studying and gaining in-depth insights into the complex behavior of multiphase multicomponent flows. Traditional CFD methods simulate multiphase/multicomponent flows by solving the macroscopic Navier-Stokes equations (NSEs) together with a proper technique to track or capture the interface among different fluids. Generally, these methods are divided into two categories: one is the interfacetracking method, which uses the Lagrangian approach to explicitly represent the interface, such as the front-tracking method [1]; and the other is the interfacecapturing method, which uses an indicator function to implicitly represent the interface in an Eulerian grid, such as the volume-of-fluid (VOF) method [2] and level set (LS) method [3, 4]. However, the front-tracking method is not suitable for handling interface breakup and coalescence, because the interface must be manually ruptured based upon some ad-hoc criteria. The VOF and LS methods require interface reconstruction or reinitialization to represent/correct the interface, which may be complex or unphysical. Physically, the interface and its dynamical behavior are the natural consequence of microscopic interactions among fluid molecules. Thus, mesoscopic level methods may be better suited to simulate complex interfacial dynamics in a multiphase/multicomponent system.

The lattice Boltzmann method (LBM), as a mesoscopic method, has been developed into an alternative to traditional CFD methods for simulating complex fluid flow problems $[5,6]$. It is a pseudo-molecular method based on particle 
distribution functions that performs microscopic operations with mesoscopic kinetic equations and reproduces macroscopic behavior [7]. The LBM has several advantages over traditional CFD methods such as the ability to be programmed on parallel computers and the ease in dealing with complex boundaries [8]. Besides, its kinetic nature provides many of the advantages of molecular dynamics, making the LBM particularly useful for simulating multiphase, multicomponent flows. A number of multiphase, multicomponent models have been proposed in the LBM community based on the Cartesian coordinate system, and they can be classified into four major types: color-gradient model $[9,10,11,12]$, phasefield-based model [13, 14, 15, 16], interparticle-potential model [17, 18, 19], and mean-field theory model [20]. Among these models, the interparticle-potential model has recently shown significant improvements in computational stability and accuracy, see, e.g., Refs. [21, 22, 23, 24, 25]. For a comprehensive review of these multiphase multicomponent models, interested readers may refer to Refs. $[8,26,27]$.

Among three-dimensional (3D) multiphase multicomponent flows, axisymmetric flows are special cases that occur when both flow geometry and initial conditions display axial symmetry. Examples of axisymmetric multiphase multicomponent flows include head on collision of two droplets [28], droplet impact on a solid surface [29], droplet formation in a co-flow microfluidic device [30], and so on. To simulate axisymmetric multiphase, multicomponent flows, a direct way is to apply a 3D multiphase/multicomponent LBM with suitable curved boundary conditions. Such a treatment, however, does not take the advantage of the axisymmetric property of the flow and usually needs large computational costs. Alternatively, several researchers have attempted to develop more effective quasi-two-dimensional (2D) LBMs for simulating axisymmetric multiphase flows. Premanath and Abraham [31] proposed the first axisymmetric multiphase LBM based on the mean-field theory model of He et al. [20]. In their method, some source terms containing density and velocity gradients were added into the microscopic evolution equations to account for axisymmetric contributions of mass, momentum, and capillary force. By introducing the source 
terms in the same fashion as Premanath and Abraham [31], Mukherjee and Abraham [32] extended the high-density-ratio model of Lee and Lin [33] to the axisymmetric case. Later, Huang et al. [34] presented a phase-field-based hybrid method for axisymmetric multiphase flows, in which they used the finite difference method for solving the axisymmetric Cahn-Hilliard equation and the multiple-relaxation-time (MRT) LBM for the NSEs. Recently, the interparticlepotential model of Shan and Chen [17] was extended by Srivastava et al. [35] to study generic axisymmetric, density-varying flows and multiphase flows. In the axisymmetric model, a source term containing velocity gradients was introduced into the lattice Boltzmann equation so that it could match the axisymmetric NSEs in the Chapman-Enskog analysis, and an axisymmetic Shan-Chen force was derived by means of a Taylor expansion of its 3D counterpart as well as a coordinate transformation. More recently, Liang et al. [36] extended their previously developed phase-field-based model [37] for the solution of axisymmetric multiphase flows. Unlike other axisymmetric multiphase LBMs, the added source terms that arise from the axisymmetric effect contain no gradients in this method, thus simplifying the computation and enhancing the computing efficiency.

As reviewed above, all types of the (Cartesian) multiphase, multicomponent LBMs have been extended to the axisymmetric versions except the colorgradient model. Compared to other multiphase multicomponent LBMs, the color-gradient model has its own advantages such as low spurious velocities, high numerical accuracy, strict mass conservation for each fluid, and good numerical stability for a broad range of fluid properties [38]. In addition, the color-gradient model has been widely employed to simulate immiscible multicomponent flow problems, in particular those in porous media and microfluidic devices [39, 40, 41, 42]. Recently, it was also extended to model the thermocapillary flows $[43,44]$ and the contact-line dynamics with the contact angle hysteresis $[45,38]$. In view of the advantages and great success of the color-gradient model, it is necessary to develop an axisymmetric version of the color-gradient LBM that allows for the solution of multicomponent flows at the computational 
cost of a $2 \mathrm{D}$ simulation.

In this work, an axisymmetric multicomponent LBM based on the colorgradient model is developed. In this method, a source term is added into the single-component collision operator so that the axisymmetric NSEs could be correctly reproduced in each single-component region. An interfacial force of axisymmetric form is derived using the concept of continuum surface force (CSF) [46] together with a coordinate transformation, and it is then incorporated into the LBM using a body force model. A recoloring algorithm proposed by Latva-Kokko and Rothman [47] is extended to axisymmetric case for producing phase segregation. In addition, the axisymmetric multicomponent LBM is implemented in a MRT framework in order to minimize spurious velocities and increase the numerical stability of solving large viscosity ratio problems $[48,49,50,38]$. The capability and accuracy of this method are tested by several typical flow cases, including simulations of a static droplet, oscillation of a viscous droplet, and breakup of a liquid thread.

\section{Theory and Mathematical Model}

In this section, an axisymmetric version of the color-gradient multicomponent LBM is presented, and it is developed on the basis of the model of Halliday and his coworkers [10, 51, 52], which is defined in a Cartesian coordinate system. There are two fluids, red and blue, considered in the color-gradient LBM. Let $f_{i}^{k}(\vec{x}, t)$ represents the particle distribution function (PDF) of the fluid $k$ in the $i$-th velocity direction at the position $\vec{x}$ and time $t$, where $k=R$ or $B$ denotes the red or blue fluid. The total distribution function is defined as $f_{i}=f_{i}^{R}+f_{i}^{B}$. The time evolution of each colored PDF is a combination of free streaming and collision:

$$
f_{i}^{k}\left(\vec{x}+\vec{e}_{i} \delta_{t}, t+\delta_{t}\right)=f_{i}^{k}(\vec{x}, t)+\Omega_{i}^{k}(\vec{x}, t),
$$

where $\vec{e}_{i}$ is the lattice velocity in the $i$ th direction, $\delta_{t}$ is the time step, and $\Omega_{i}^{k}$ is the collision operator. The collision operator consists of three separate 
parts $[11,45]$ :

$$
\Omega_{i}^{k}=\left(\Omega_{i}^{k}\right)^{(3)}\left[\left(\Omega_{i}^{k}\right)^{(1)}+\left(\Omega_{i}^{k}\right)^{(2)}\right],
$$

where $\left(\Omega_{i}^{k}\right)^{(1)}$ is the single-component collision operator, $\left(\Omega_{i}^{k}\right)^{(2)}$ is the perturbation operator which contributes to the mixed interfacial region and generates an interfacial tension, and $\left(\Omega_{i}^{k}\right)^{(3)}$ represents the recoloring operator which mimics the phase segregation and keeps the interface sharp.

The single-component collision operator $\left(\Omega_{i}^{k}\right)^{(1)}$ is designed to recover the correct macroscopic equations of incompressible axisymmetric flows in each single-component region. For the axisymmetric flows with an axis in the $z$ direction, the continuity and momentum equations in the cylindrical coordinates, in absence of external forces are given by $[35,36]$

$$
\partial_{\alpha} u_{\alpha}=-u_{r} / r
$$

and

$\rho\left(\partial_{t} u_{\alpha}+u_{\beta} \partial_{\beta} u_{\alpha}\right)=-\partial_{\alpha} p+\partial_{\beta}\left[\mu\left(\partial_{\beta} u_{\alpha}+\partial_{\alpha} u_{\beta}\right)\right]+\frac{\mu\left(\partial_{r} u_{\alpha}+\partial_{\alpha} u_{r}\right)}{r}-\frac{2 \mu u_{r}}{r^{2}} \delta_{\alpha r}$,

respectively, where $\alpha, \beta$ indicate the $r$ or $z$ component, and $r$ is the coordinate in radial direction; $u_{\alpha}$ is the component of velocity in the $\alpha$ direction; $p$ is the pressure; $\rho$ and $\mu$ are the density and dynamic viscosity of the fluid mixture (or color-blind fluid); and $\delta_{\alpha \beta}$ is the Kronecker delta with two indices. In Eqs.(3) and (4) we assume that the flows considered have no swirl, i.e. the azimuthal velocity $u_{\theta}=0$, and that both red and blue fluids have equal densities for the sake of simplicity. With the aid of the continuity equation (3), one can rewrite Eq.(4) as [53]

$$
\begin{array}{r}
\rho\left[\partial_{t} u_{\alpha}+\partial_{\beta}\left(u_{\alpha} u_{\beta}\right)\right]=-\partial_{\alpha} p+\partial_{\beta}\left[\mu\left(\partial_{\beta} u_{\alpha}+\partial_{\alpha} u_{\beta}\right)\right] \\
+\frac{\mu\left(\partial_{r} u_{\alpha}+\partial_{\alpha} u_{r}\right)}{r}-\frac{2 \mu u_{r}}{r^{2}} \delta_{\alpha r}-\frac{\rho u_{\alpha} u_{r}}{r} .
\end{array}
$$

The term on the right-hand side of Eq.(3) and the last three terms on the righthand side of Eq.(5) arise from the cylindrical polar coordinates, and they are hereafter referred to as the additional terms. In order to recover these terms, we 
follow the previous works $[54,34,35]$ and define the single-component collision operator as

$$
\left(\Omega_{i}^{k}\right)^{(1)}=-\frac{1}{\tau}\left[f_{i}^{k}(\vec{x}, t)-f_{i}^{k, e q}(\vec{x}, t)\right]+\delta_{t} h_{i}^{k}\left(\vec{x}+\vec{e}_{i} \delta_{t} / 2, t+\delta_{t} / 2\right),
$$

which uses the standard Bhatnagar-Gross-Krook (BGK) approximation where the $\mathrm{PDF} f_{i}^{k}$ is relaxed towards its equilibrium distribution function $f_{i}^{k, e q}$ with a single relaxation time $\tau$. For the two-dimensional 9-velocity (D2Q9) model [55], the lattice velocity $\vec{e}_{i} \equiv\left(e_{i r}, e_{i z}\right)$ is defined as $\vec{e}_{0}=(0,0), \vec{e}_{1,3}=( \pm c, 0), \vec{e}_{2,4}=$ $(0, \pm c), \vec{e}_{5,7}=( \pm c, \pm c)$, and $\vec{e}_{6,8}=(\mp c, \pm c)$, where $c=\delta_{x} / \delta_{t}$ is the lattice speed and $\delta_{x}$ the lattice spacing. The equilibrium distribution function is obtained by a Taylor expansion of Maxwell-Boltzmann distribution with respect to the velocity $\vec{u}$ :

$$
f_{i}^{k, e q}=\rho_{k} w_{i}\left[1+\frac{\vec{e}_{i} \cdot \vec{u}}{c_{s}^{2}}+\frac{\left(\vec{e}_{i} \cdot \vec{u}\right)^{2}}{2 c_{s}^{4}}-\frac{\vec{u}^{2}}{2 c_{s}^{2}}\right],
$$

where $\rho_{k}$ is the local density of the fluid $k, c_{s}=c / \sqrt{3}$ is the speed of sound, and the weight coefficients $w_{i}$ are given by $w_{0}=4 / 9, w_{1-4}=1 / 9$ and $w_{5-8}=1 / 36$. Conservation of mass for each fluid and total momentum conservation require

$$
\begin{gathered}
\rho_{k}=\sum_{i} f_{i}^{k}=\sum_{i} f_{i}^{k, e q}, \\
\rho u_{\alpha}=\sum_{k} \sum_{i} f_{i}^{k} e_{i \alpha}=\sum_{k} \sum_{i} f_{i}^{k, e q} e_{i \alpha},
\end{gathered}
$$

where $\rho=\sum_{k} \rho_{k}$.

The source term $h_{i}^{k}$ in Eq.(6) is introduced to recover the additional terms in the continuity and momentum equations [i.e., Eqs.(3) and (5)], and it is given by

$$
h_{i}^{k}=-w_{i} \frac{\rho_{k} u_{r}}{r}+\frac{1}{c_{s}^{2}} w_{i} e_{i \alpha} H_{\alpha}^{k}
$$

with

$$
H_{\alpha}^{k}=\frac{\nu \rho_{k}\left(\partial_{r} u_{\alpha}+\partial_{\alpha} u_{r}\right)}{r}-\frac{2 \nu \rho_{k} u_{r}}{r^{2}} \delta_{\alpha r}-\frac{\rho_{k} u_{\alpha} u_{r}}{r},
$$

where $\nu=\mu / \rho$ is the kinematic viscosity of the fluid mixture.

Using the Chapman-Enskog multiscale expansion, the continuity and momentum equations can be exactly derived from Eqs.(1), (2) and (6)-(11) in 
absence of the perturbation and recoloring operators, where the pressure and the fluid viscosity are given by (see Appendix A for the derivation)

$$
\begin{aligned}
p & =\rho c_{s}^{2}, \\
\mu & =\rho c_{s}^{2}\left(\tau-\frac{1}{2}\right) \delta_{t} .
\end{aligned}
$$

According to Eq.(6), all of the terms in $h_{i}^{k}$, given by Eqs.(10) and (11), take their values at the position $\vec{x}+\vec{e}_{i} \delta_{t} / 2$ and time $t+\delta_{t} / 2$, which is known as centered scheme. In order to avoid the implicitness of the evolution equations, the source term is simply evaluated by

$$
h_{i}^{k}\left(\vec{x}+\vec{e}_{i} \delta_{t} / 2, t+\delta_{t} / 2\right)=h_{i}^{k}(\vec{x}, t)
$$

in the practical simulations, as previously done in Refs. $[56,57,34,35]$. It is shown in Appendix B that such a simple treatment allows us to accurately account for axisymmetric contributions in the continuity and momentum equations, consistent with the previous findings in single-phase and multiphase flow simulations [58, 56, 57, 34, 35].

To allow for unequal viscosities of the two fluids, we determine the viscosity of the fluid mixture by a harmonic mean $[59,41]$

$$
\frac{1}{\mu\left(\rho^{N}\right)}=\frac{1+\rho^{N}}{2 \mu_{R}}+\frac{1-\rho^{N}}{2 \mu_{B}},
$$

where $\mu_{k}(k=R$ or $B)$ is the dynamic viscosity of fluid $k$; and $\rho^{N}$ is the color function (or phase-field function), which is used to describe the spatial distribution of the two fluids and is defined as

$$
\rho^{N}(\vec{x}, t)=\frac{\rho_{R}(\vec{x}, t)-\rho_{B}(\vec{x}, t)}{\rho_{R}(\vec{x}, t)+\rho_{B}(\vec{x}, t)}, \quad-1 \leq \rho^{N} \leq 1 .
$$

It has been shown that the choice of Eq.(15) can ensure a constant viscosity stress across the interface, resulting into a higher accuracy than other choices [41].

In the perturbation step, the continuum surface force (CSF) model is used to model the interfacial tension, which has been demonstrated to greatly reduce spurious velocities and improve the isotropy of the interface $[10,51,60]$. In the 
CSF model, a volume-distributed interfacial force $\vec{f}_{s}$ is added in the momentum equation to induce the local stress jump across the interface. The interfacial force acts centripetally normal to the local interface with a magnitude proportional to the gradient of the color function, and its expression in 3D is

$$
\vec{f}_{s}(\vec{x}, t)=-\frac{1}{2} \sigma \nabla \cdot\left(\frac{\vec{n}}{|\vec{n}|}\right) \nabla \rho^{N},
$$

where $\sigma$ is an interfacial tension parameter, and $\vec{n}$ is the interface normal vector defined by $\vec{n}=\nabla \rho^{N}$.

In the axisymmetric case, there is an extra term in the interfacial force $\vec{f}_{s}$, that is

$$
\overrightarrow{f_{s}}(\vec{x}, t)=-\frac{1}{2} \sigma \nabla_{c} \cdot\left(\frac{\nabla_{c} \rho^{N}}{\left|\nabla_{c} \rho^{N}\right|}\right) \nabla_{c} \rho^{N}-\frac{1}{2} \sigma \frac{\partial_{r} \rho^{N}}{r\left|\nabla_{c} \rho^{N}\right|} \nabla_{c} \rho^{N},
$$

where $\nabla_{c}$ is the gradient in the cylindrical coordinates given by $\nabla_{c}=\left(\partial_{r}, \partial_{z}\right)$. It is noted in the above equation that the first term on the right-hand side is the one adopted by the color-gradient model in two dimensions, and that the last term is the extra term responsible for the three dimensionality. Following the previous works $[51,45]$, the $2 \mathrm{D}$ curvature $\nabla_{c} \cdot\left(\frac{\nabla_{c} \rho^{N}}{\mid \nabla_{c} \rho^{N}}\right)$ in Eq.(18) can be rewritten as

$$
\nabla_{c} \cdot\left(\frac{\nabla_{c} \rho^{N}}{\left|\nabla_{c} \rho^{N}\right|}\right)=\hat{n}_{r} \hat{n}_{z}\left(\partial_{r} \hat{n}_{z}+\partial_{z} \hat{n}_{r}\right)-\hat{n}_{r}^{2} \partial_{z} \hat{n}_{z}-\hat{n}_{z}^{2} \partial_{r} \hat{n}_{r}
$$

where $\hat{n}_{\alpha}=\frac{\partial_{\alpha} \rho^{N}}{\sqrt{\left(\partial_{r} \rho^{N}\right)^{2}+\left(\partial_{z} \rho^{N}\right)^{2}}}$.

The interfacial force Eq.(18) can be incorporated into the LBM through different schemes. In the present study, the force model of Guo et al. [61] is employed for its high accuracy in modeling a spatially varying body force and capability in reducing effectively spurious velocities. According to Guo et al., the perturbation operator responsible for generating the interfacial tension is expressed by [45]

$$
\left(\Omega_{i}^{k}\right)^{(2)}=A_{k}\left(1-\frac{1}{2 \tau}\right) w_{i}\left(\frac{e_{i \alpha}-u_{\alpha}}{c_{s}^{2}}+\frac{e_{i \beta} u_{\beta}}{c_{s}^{4}} e_{i \alpha}\right) f_{s \alpha}(\vec{x}, t) \delta_{t},
$$

where $A_{k}$ is the fraction of interfacial tension contributed by the fluid $k$, and satisfies $\sum_{k} A_{k}=1$. In the presence of the interfacial tension force, the velocity 
should be re-defined to correctly recover the Navier-Stokes equations, i.e.,

$$
\rho(\vec{x}, t) u_{\alpha}(\vec{x}, t)=\sum_{k} \sum_{i} f_{i}^{k}(\vec{x}, t) e_{i \alpha}+\frac{1}{2} f_{s \alpha}(\vec{x}, t) \delta_{t} .
$$

Although the perturbation operator generates an interfacial tension, it does not ensure the immiscibility of both fluids. To promote phase segregation and maintain the interface, the recoloring algorithm proposed by Latva-Kokko and Rothman [47] is applied. This algorithm allows the red and blue fluids to mix moderately at the tangent of the interface, and at the same time keeps the color distribution symmetric with respect to the color gradient. Thus, it can further reduce spurious velocities and remove the lattice pinning problem arising in the original recoloring operator of Gunstensen et al. [9]. By replacing the 3D gradient $\nabla$ with its axisymmetric counterpart $\nabla_{c}$, one can obtain the recoloring operator in the axisymmetric case, which reads as

$$
\begin{aligned}
& \left(\Omega_{i}^{R}\right)^{(3)}\left(f_{i}^{R \ddagger}\right)=\frac{\rho_{R}}{\rho} f_{i}^{\dagger}(\vec{x}, t)+\beta \frac{\rho_{R} \rho_{B}}{\rho} w_{i} \frac{\vec{e}_{i} \cdot \nabla_{c} \rho^{N}}{\left|\vec{e}_{i}\right|\left|\nabla_{c} \rho^{N}\right|}, \\
& \left(\Omega_{i}^{B}\right)^{(3)}\left(f_{i}^{B \dagger}\right)=\frac{\rho_{B}}{\rho} f_{i}^{\dagger}(\vec{x}, t)-\beta \frac{\rho_{R} \rho_{B}}{\rho} w_{i} \frac{\vec{e}_{i} \cdot \nabla_{c} \rho^{N}}{\left|\vec{e}_{i}\right|\left|\nabla_{c} \rho^{N}\right|},
\end{aligned}
$$

where $f_{i}^{\dagger}$ is the post-perturbation value of the total distribution function; $f_{i}^{R \ddagger}$ and $f_{i}^{B \ddagger}$ are the post-segregation (recolored) distribution functions of the red and blue fluids, respectively; $\beta$ is a free parameter associated with the interface thickness and takes a value between zero and unity. In this study, $\beta$ is taken as 0.7 to maintain a steady interface [52], which corresponds to an interface thickness of 4 to 5 lattices. In addition, a previous study also showed that this choice is necessary to reproduce correct droplet behavior [12].

In contrast to the BGK approximation, the multiple-relaxation-time (MRT) model is able to enhance numerical stability and reduce spurious velocities at the phase interface by tuning the adjustable relaxation parameters. Thus, it has been widely used in various multiphase/multicomponent LBMs instead of the BGK approximation [62, 63, 48, 49, 37, 38]. With the MRT model, the singlecomponent collision operator and the perturbation operator can be written as

$$
\left(\Omega_{i}^{k}\right)^{(1)}=-\sum_{j}\left(\mathbf{M}^{-1} \mathbf{S}\right)_{i j}\left[m_{j}^{k}(\vec{x}, t)-m_{j}^{k, e q}(\vec{x}, t)\right]+\delta_{t} h_{i}^{k}(\vec{x}, t),
$$




$$
\left(\Omega_{i}^{k}\right)^{(2)}=-\sum_{j}\left[\mathbf{M}^{-1}\left(\mathbf{I}-\frac{1}{2} \mathbf{S}\right)\right]_{i j} F_{j}^{k}(\vec{x}, t),
$$

where the transformation matrix $\mathbf{M}$ is given by [64]

$$
\mathbf{M}=\left(\begin{array}{ccccccccc}
1 & 1 & 1 & 1 & 1 & 1 & 1 & 1 & 1 \\
-4 & -1 & -1 & -1 & -1 & 2 & 2 & 2 & 2 \\
4 & -2 & -2 & -2 & -2 & 1 & 1 & 1 & 1 \\
0 & 1 & 0 & -1 & 0 & 1 & -1 & -1 & 1 \\
0 & -2 & 0 & 2 & 0 & 1 & -1 & -1 & 1 \\
0 & 0 & 1 & 0 & -1 & 1 & 1 & -1 & -1 \\
0 & 0 & -2 & 0 & 2 & 1 & 1 & -1 & -1 \\
0 & 1 & -1 & 1 & -1 & 0 & 0 & 0 & 0 \\
0 & 0 & 0 & 0 & 0 & 1 & -1 & 1 & -1
\end{array}\right)
$$

Through the transformation matrix $\mathbf{M}$, the $\operatorname{PDF} f_{i}^{k}$ and its equilibrium distribution $f_{i}^{k, e q}$ can be projected onto the moment space as $m_{i}^{k}=\sum_{j} \mathbf{M}_{i j} f_{j}^{k}$ and $m_{i}^{k, e q}=\sum_{j} \mathbf{M}_{i j} f_{j}^{k, e q}$. The resulting equilibrium distribution function in the moment space is given by

$$
\begin{gathered}
\mathbf{m}^{k, e q}=\left(m_{0}^{k, e q}, m_{1}^{k, e q}, m_{2}^{k, e q}, \cdots, m_{8}^{k, e q}\right)^{\mathrm{T}} \\
=\rho_{k}\left(1,-2+3\left(u_{r}^{2}+u_{z}^{2}\right), 1-3\left(u_{r}^{2}+u_{z}^{2}\right), u_{r},-u_{r}, u_{z},-u_{z}, u_{r}^{2}-u_{z}^{2}, u_{r} u_{z}\right)^{\mathrm{T}}(26)
\end{gathered}
$$

In Eqs.(23) and (24), $\mathbf{I}$ is a $9 \times 9$ identity matrix and $\mathbf{S}$ is a non-negative diagonal matrix defined by

$$
\mathbf{S}=\operatorname{diag}\left(s_{0}, s_{1}, s_{2}, s_{3}, s_{4}, s_{5}, s_{6}, s_{7}, s_{8}\right),
$$

where the element $s_{i}$ represent the inverse of the relaxation time for the transformed PDF $m_{i}^{k}$ as it is relaxed to the equilibrium distribution function in the moment space, $m_{i}^{k, e q}$. The parameters $s_{0}, s_{3}$ and $s_{5}$ correspond to the conserved moments (i.e., density and momentum) and have no effect on the derivation of the NSEs [65]. For simplicity, we choose $s_{0}=s_{3}=s_{5}=0 . s_{1}$ determines the bulk viscosity $\zeta$ through

$$
\zeta=\left(\frac{1}{s_{1}}-\frac{1}{2}\right) c_{s}^{2} \delta_{t}
$$


and it is considered as an adjustable parameter since the binary fluids are incompressible. $s_{7}$ and $s_{8}$ are related to the viscosity of fluid mixture by

$$
s_{7}=s_{8}=\frac{1}{\tau}, \quad \text { and } \mu=\rho c_{s}^{2}\left(\tau-\frac{1}{2}\right) \delta_{t} .
$$

Besides, symmetry requires that $s_{4}=s_{6}$. Consequently, three independent parameters $s_{1}, s_{2}$ and $s_{4}\left(=s_{6}\right)$ can be freely adjusted to enhance the stability of MRT model [66, 50,62,63]. Following the guidelines and suggestions in Ref. [64], we choose $s_{1}=1.63, s_{2}=1.14$, and $s_{4}=1.92$ in this study. It was also demonstrated that such a choice can effectively suppress unphysical spurious velocities in the vicinity of the contact line, resulting in an increased numerical accuracy in simulating contact angles [50]. The MRT forcing term $\mathbf{F}^{k}$ is given by [63]

$$
\begin{aligned}
\mathbf{F}^{k}= & \left(F_{0}^{k}, F_{1}^{k}, F_{2}^{k}, \cdots, F_{8}^{k}\right)^{\mathrm{T}}=A_{k}\left(0,6\left(u_{r} f_{s r}+u_{z} f_{s z}\right),-6\left(u_{r} f_{s r}+u_{z} f_{s z}\right),\right. \\
& \left.f_{s r},-f_{s r}, f_{s z},-f_{s z}, 2\left(u_{r} f_{s r}-u_{z} f_{s z}\right), u_{r} f_{s z}+u_{z} f_{s r}\right)^{\mathrm{T}} .
\end{aligned}
$$

Finally, we note in the MRT framework that, the recoloring operator is kept the same as the one in the BGK framework, as previously given by Eq.(22), because it does not contain any terms related to the relaxation time.

\section{Numerical Validations}

In this section, the axisymmetric color-gradient LBM proposed in Sect.2 is validated by three typical cases, namely static droplet test, oscillation of a viscous droplet, and breakup of a liquid thread. In each of the simulations below, $r=0$ represents the axis of symmetry, and the singularity will occur at $r=0$ because of the terms containing $r^{-1}[54,36]$. To avoid the singularity, we set the first lattice line at $r=0.5 \delta_{x}$ and apply the symmetry boundary condition to a ghost lattice line positioned at $r=-0.5 \delta_{x}$ (see Fig.1):

$$
\begin{array}{lll}
f_{1}^{R \ddagger}(P)=f_{3}^{R \ddagger}(Q), & f_{5}^{R \ddagger}(P)=f_{6}^{R \ddagger}(Q), & f_{8}^{R \ddagger}(P)=f_{7}^{R \ddagger}(Q), \\
f_{1}^{B \ddagger}(P)=f_{3}^{B \ddagger}(Q), & f_{5}^{B \ddagger}(P)=f_{6}^{B \ddagger}(Q), & f_{8}^{B \ddagger}(P)=f_{7}^{B \ddagger}(Q),
\end{array}
$$




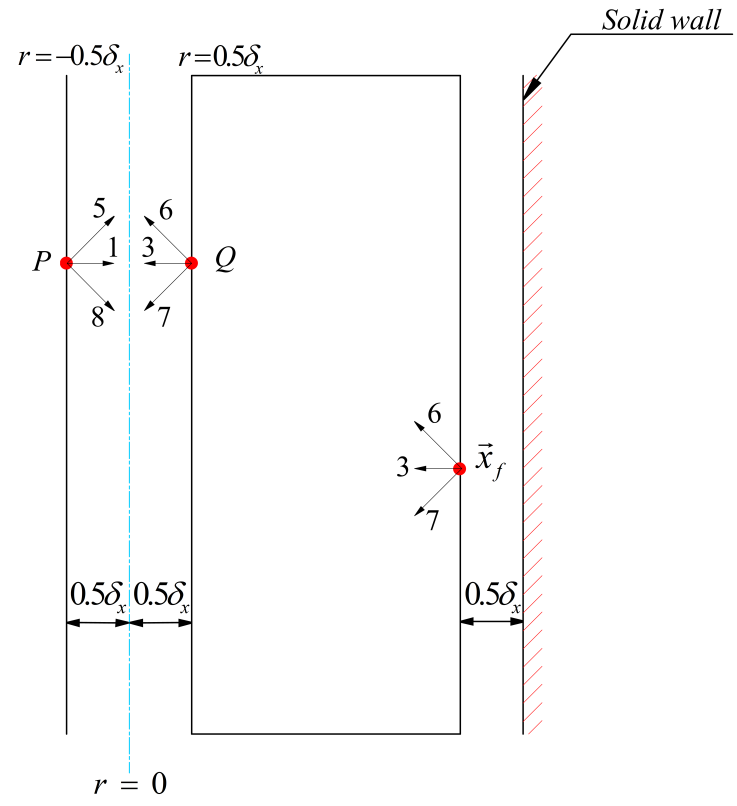

Figure 1: (Color Online) Schematic diagram of the computational geometry and setup of the boundary conditions.

where $Q$ is an arbitrary node at the first fluid line, and $P$ is the symmetric ghost node of $Q$. For the solid wall, no-slip boundary condition is enforced using the halfway bounce-back scheme [67], which means the particles that hit the solid wall, then simply return back in the opposite direction where they came from. Specifically, as shown in Fig.1, the unknown PDFs at the fluid node $\vec{x}_{f}$ adjacent to the solid wall are determined by

$$
\begin{aligned}
& f_{3}^{R}\left(\vec{x}_{f}, t+\delta_{t}\right)=f_{1}^{R \ddagger}\left(\vec{x}_{f}, t\right), f_{6}^{R}\left(\vec{x}_{f}, t+\delta_{t}\right)=f_{8}^{R \ddagger}\left(\vec{x}_{f}, t\right), f_{7}^{R}\left(\vec{x}_{f}, t+\delta_{t}\right)=f_{5}^{R \ddagger}\left(\vec{x}_{f}, t\right), \\
& f_{3}^{B}\left(\vec{x}_{f}, t+\delta_{t}\right)=f_{1}^{B \ddagger}\left(\vec{x}_{f}, t\right), f_{6}^{B}\left(\vec{x}_{f}, t+\delta_{t}\right)=f_{8}^{B \ddagger}\left(\vec{x}_{f}, t\right), f_{7}^{B}\left(\vec{x}_{f}, t+\delta_{t}\right)=f_{5}^{B \ddagger}\left(\vec{x}_{f}, t\right) .
\end{aligned}
$$

The partial derivatives in the source term $h_{i}^{k}$ and the interfacial force $\vec{f}_{s}$ should be evaluated via suitable difference schemes. To minimize the discretization errors, the fourth-order isotropic finite-difference scheme,

$$
\partial_{\alpha} \psi(\vec{x})=\frac{1}{c_{s}^{2} \delta_{t}} \sum_{i} w_{i} \psi\left(\vec{x}+\vec{e}_{i} \delta_{t}\right) e_{i \alpha},
$$


is used to evaluate the derivatives of a variable $\psi$ at $\vec{x} \neq \vec{x}_{f}$; whereas at the fluid node $\vec{x}_{f}$ we impose the derivative terms to be zero in the evaluation of the interfacial force, and use the second-order difference schemes to evaluate the derivative terms in $h_{i}^{k}$, i.e.

$$
\begin{aligned}
& \partial_{r} \psi\left(\vec{x}_{f}\right)=-\frac{1}{3 \delta_{x}}\left[3 \psi\left(\vec{x}_{f}\right)+\psi\left(\vec{x}_{f}+\vec{e}_{3} \delta_{t}\right)\right], \\
& \partial_{z} \psi\left(\vec{x}_{f}\right)=\frac{1}{2 \delta_{x}}\left[\psi\left(\vec{x}_{f}+\vec{e}_{2} \delta_{t}\right)-\psi\left(\vec{x}_{f}+\vec{e}_{4} \delta_{t}\right)\right],
\end{aligned}
$$

which is obtained on the basis of the zero velocity condition at the solid wall. In addition, it is worth mentioning that, in what follows, all of the simulation results are obtained by our proposed LBM in Sect.2 unless otherwise noted.

\subsection{Static droplet test}

The static droplet test represents a traditional benchmark of two-phase flow models. It consists of a 'spherical' droplet (red fluid) initially located at the centre of the lattice domain with $100 \times 200$ lattices in the $r z$-plane. The boundary conditions for both $f_{i}^{R}$ and $f_{i}^{B}$ are periodic in the $z$-direction and the right boundary is the solid wall where the no-slip boundary condition is imposed. According to the Laplace's law, when the system reaches the equilibrium state, the pressure difference between the interior and exterior of the droplet $\Delta p$ is related to the interfacial tension $\sigma$ by

$$
\Delta p=\frac{2 \sigma}{R_{D}},
$$

where $R_{D}$ is the radius of the droplet. Fig.2 shows the pressure difference $\Delta p$ against $R_{D}^{-1}$ using the following parameters: $\rho_{R}=\rho_{B}=1, \mu_{R}=0.3$, $\mu_{B}=0.03$, and $\sigma=0.01$. It can be found that our LBM results (represented by hollow circles) are in excellent agreement with the Laplace's law (represented by the solid line). Based on the recoloring operator Eq.(22), one can derive an analytical expression for the equilibrium interface profile:

$$
\rho^{N}(r, z)=\tanh \left(\frac{R_{D}-\sqrt{\left(r-r_{c}\right)^{2}+\left(z-z_{c}\right)^{2}}}{\xi}\right),
$$




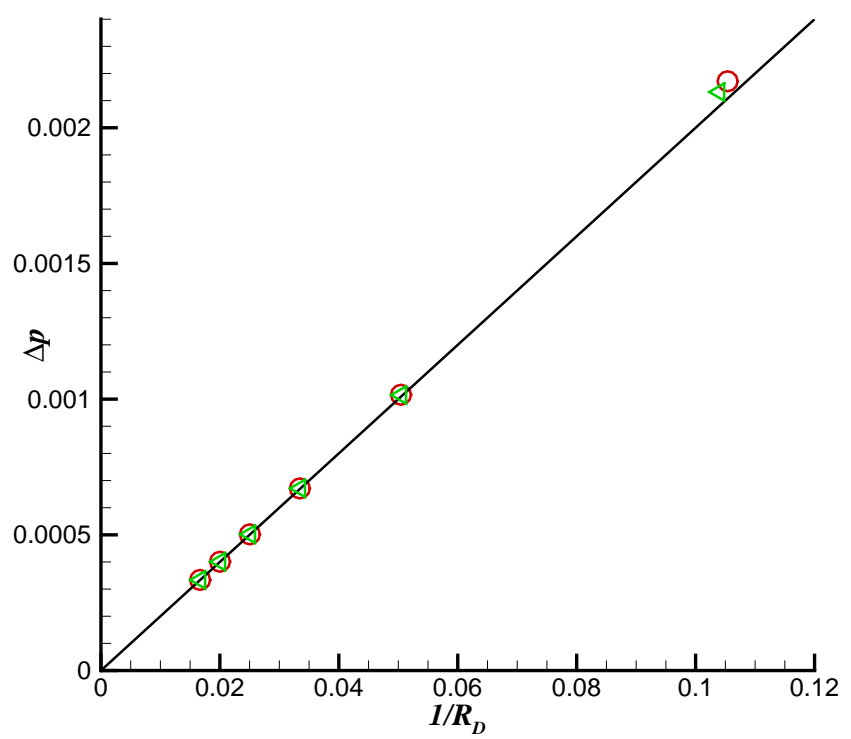

Figure 2: (Color Online) Comparison of the LBM results (represented by discrete symbols) with the Laplace's law (represented by the solid line) for pressure jump across a static droplet interface. Note that the red circles represent the simulation results from the color-gradient LBM proposed in Sect.2, while the green triangles represent the simulation results from the Li-based model that is presented in Appendix B. 


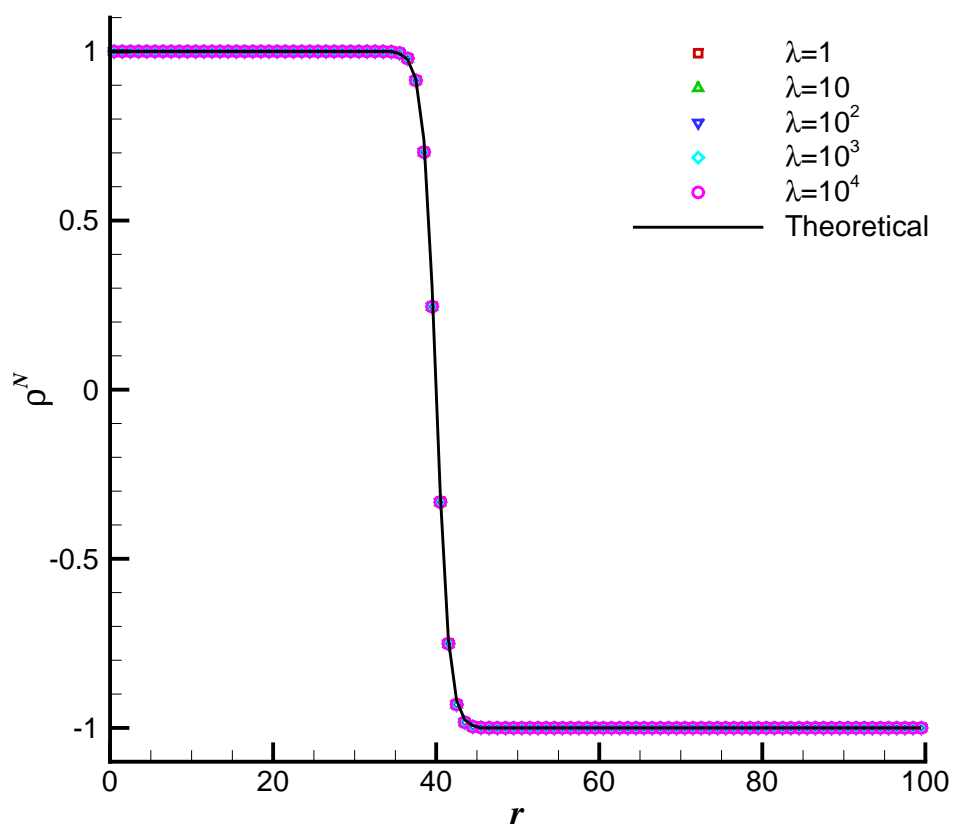

Figure 3: (Color Online) The profiles of the color function at different values of $\lambda$ for a static droplet with $R_{D}=40$. The discrete symbols represent the simulation results of the present LBM and the solid line is the theoretical profile given by Eq.(36). 
where $r_{c}$ and $z_{c}$ are the coordinates of the centre of the droplet, and $\xi$ is a measure of the interface thickness related to the segregation parameter $\beta$ by $\xi=1 /(6 k \beta)[68]$. Here $k$ is a geometric constant that is determined by

$$
2 k=\sum_{i} \frac{w_{i} e_{i \alpha} e_{i \beta}}{\left|\vec{e}_{i}\right|} .
$$

From Eq.(37), one can easily obtain $k=\frac{1}{9}+\frac{1}{18 \sqrt{2}} \approx 0.1504$ for the D2Q9 lattice. Next, we conduct a series of LBM simulations for $R_{D}=40$ over a broad range of viscosity ratios $\left(\lambda=\frac{\mu_{R}}{\mu_{B}}\right)$, and compare the simulated equilibrium interface profiles with the analytical one given by Eq.(36). In these simulations, all the parameters are kept the same as those used in Fig.2 except $\mu_{B}$, which is varied to obtain different viscosity ratios. Fig.3 displays the color function $\rho^{N}$ as a function of the distance from the droplet centre for $\lambda=\left\{1,10,10^{2}, 10^{3}, 10^{4}\right\}$. It can be clearly seen that the LBM simulations are stable for the viscosity ratios up to $10^{4}$, which is much higher than the highest viscosity ratio that other multicomponent LBMs can achieve, e.g., the highest values of the viscosity ratio are only on the order of 6 and tens for the interparticle-potential model and the free-energy model, respectively [27]. Also, the equilibrium interface profiles are all in good agreement with the analytical solution, indicating that our axisymmetric multicomponent LBM can correctly model and capture phase interface. It can be seen from the analytical expression of interface profile that the interface thickness is only determined by the segregation parameter $\beta$ and is independent of the choice of BGK or MRT collision models (provided that both models produce stable numerical results). This is confirmed by our numerical results presented in Fig.4, where (a) compares the MRT results of $\lambda=10$ and $10^{4}$ with the analytical predictions from Eq.(36) for $\beta=1$ (which leads to a thinner interface than $\beta=0.7$ ), and (b) compares the numerical results obtained by the BGK model with those by the MRT model for $\beta=0.7$ and $\lambda=10$. The present color-gradient LBM is a diffuse-interface model, which requires the Cahn number $C n=\xi / R_{D} \ll 1$ to recover a sharp-interface limit. Since $\xi=\frac{1}{6 k \beta}=\frac{1}{6 \times 0.1504 \times 0.7}=1.583$ is a constant when $\beta$ is fixed at 0.7 (for a larger $\beta$, e.g. $\beta=1$, the interface cannot be accurately described because of 


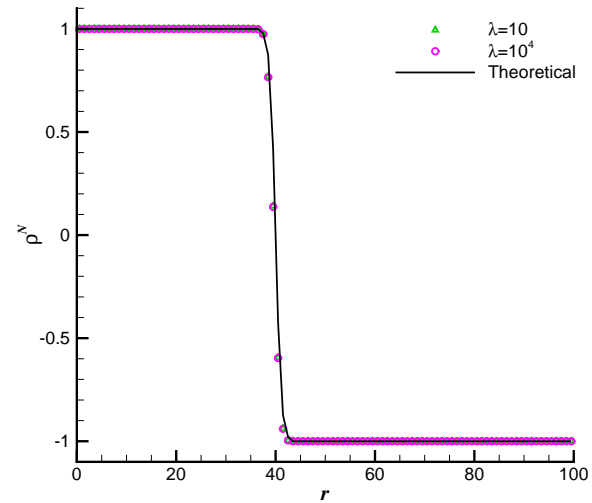

(a)

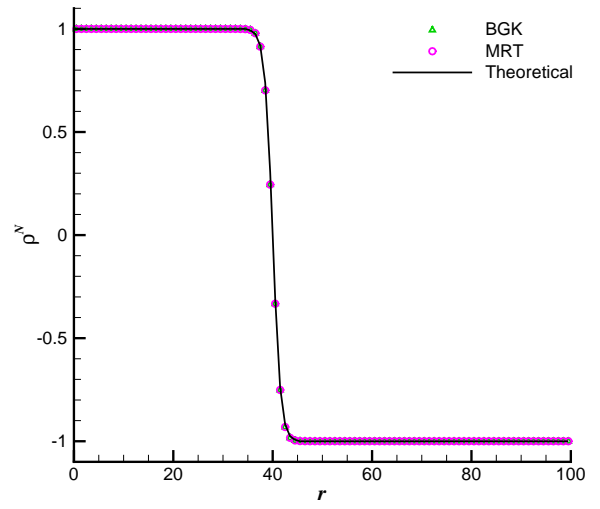

(b)

Figure 4: (Color Online) The profiles of the color function obtained by (a) the MRT model at $\beta=1$ and $\lambda=\left\{10,10^{4}\right\}$ and (b) the BGK and MRT models at $\beta=0.7$ and $\lambda=10$, for a static droplet with $R_{D}=40$. The discrete symbols represent the simulation results and the solid lines are the theoretical profiles given by Eq.(36).

insufficient number of lattice grids across the interface, which can be seen in Fig.4(a) and Ref. [52]), small value of $C n$ can be only achieved by increasing the grid number of droplet size or computational domain, which will largely increase the computing cost. To strike a balance between the computing cost and accuracy, we take $R_{D}=40$ lattices so that $C n=0.0396$, which was previously demonstrated small enough to provide satisfactory predictions of the droplet dynamics in color-gradient LBMs [41, 42, 38, 69].

A numerical artifact observed in many numerical methods is the presence of spurious velocities at the phase interface. Spurious velocities are smallamplitude artificial velocity fields arising from an imbalance between discretized forces in the interfacial region. Spurious velocities on one hand may prevent the system from reaching a true equilibrium state, and on the other hand, they can sometimes be as large as the characteristic velocity of the flow problem, leading to numerical instability and/or contamination of physical velocities. Several attempts have been made to identify the cause of spurious velocities and to reduce their magnitude, see Refs. [51, 10] and a general review by Connington and Lee [70]. However, none of the existing color-gradient LBMs can elimi- 
nate the spurious velocities to roundoff. This is also true for the present LBM. Table 1 shows the maximum spurious velocities $\left(|\vec{u}|_{\max }\right)$ at various viscosity ratios, where the values of $|\vec{u}|_{\max }$ are magnified by $10^{5}$ times. It is seen that the maximum spurious velocities increase with the viscosity ratio, and that all of them are on the order of $10^{-4}$ or even smaller, comparable to those produced by the original 2D color-gradient model (see the bottom row in Table 1). Also, the present spurious velocities are much smaller than those obtained with the commonly-used interparticle-potential model [71, 40].

Table 1: The maximum spurious velocities $\left(|\vec{u}|_{\max }\right)$ at various viscosity ratios for $R_{D}=40$ and $\mu_{R}=0.3$.

\begin{tabular}{cc|ccccc}
\hline \hline$\lambda$ & 1 & 10 & $10^{2}$ & $10^{3}$ & $10^{4}$ \\
\hline \multirow{2}{*}{$|\vec{u}|_{\max } \times 10^{5}$} & Axisymmetic LBM & 0.223 & 0.229 & 1.327 & 8.533 & 26.548 \\
& Original 2D LBM & 0.204 & 0.206 & 0.752 & 5.435 & 19.952 \\
\hline
\end{tabular}

\subsection{Oscillation of a viscous droplet}

Droplet oscillation is often used to assess whether an axisymmetric multiphase/multicomponent model is able to simulate dynamic problems. A droplet (red fluid) that is slightly deformed to be an axisymmetric ellipsoid, is immersed in a second viscous fluid (blue fluid). Upon release, the ellipsoidal droplet starts to oscillate due to the imbalanced interfacial tension forces. For the droplet oscillation, Miller and Scriven [72] derived an analytical solution for the oscillation frequency of the $n$th mode,

$$
\omega_{n}=\omega_{n}^{*}-\frac{1}{2} a \sqrt{\omega_{n}^{*}}+\frac{1}{4} a^{2},
$$

where $\omega_{n}^{*}$ is Lamb's natural resonance frequency,

$$
\omega_{n}^{*}=\sqrt{\frac{n(n+1)(n-1)(n+2)}{R_{D}^{3}\left[n \rho_{B}+(n+1) \rho_{R}\right]}} \sigma,
$$

and $R_{D}$ is the radius of the droplet at equilibrium. In Eq.(38), the last two terms represent the corrections to $\omega_{n}^{*}$ due to viscous effects, and the parameter 

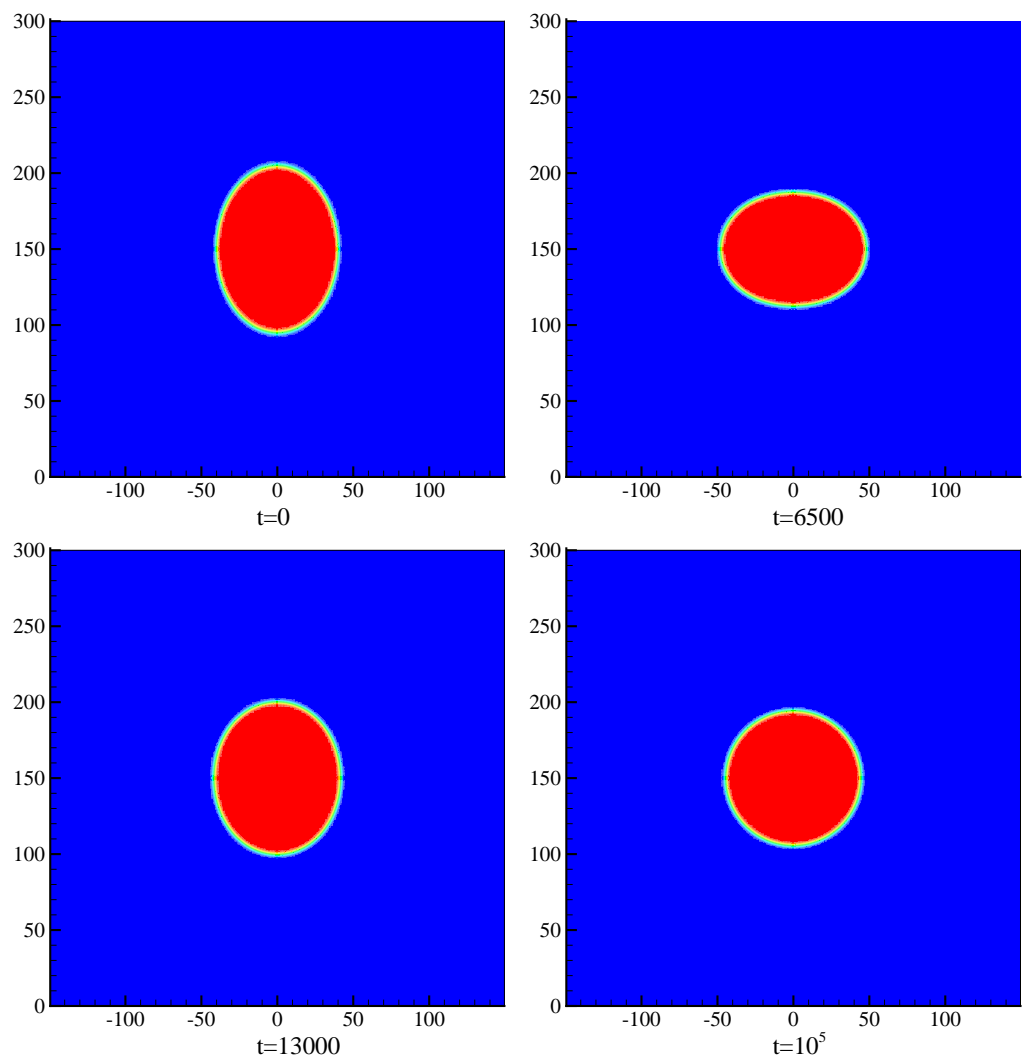

Figure 5: (Color Online) Evolution of the shape of an oscillating droplet for $R_{r}=40, R_{z}=55$, $\mu_{R}=2 \times 10^{-2}, \mu_{B}=2 \times 10^{-3}$, and $\sigma=5 \times 10^{-3}$. 


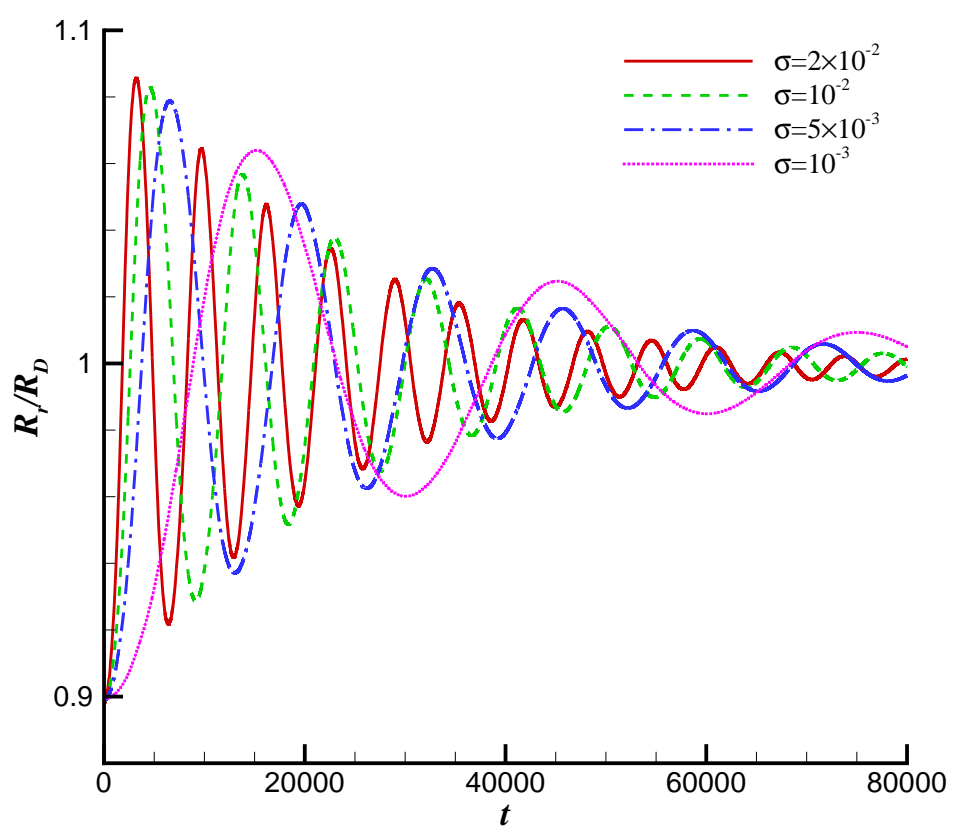

Figure 6: (Color Online) Time evolution of the half-axis length $R_{r}$ at different values of $\sigma$ for $R_{r}=40, R_{z}=55, \mu_{R}=2 \times 10^{-2}$, and $\mu_{B}=2 \times 10^{-3}$. Note that the half-axis length $R_{r}$ is normalized by the equilibrium radius $R_{D}$. 
$a$ is given by

$$
a=\frac{(2 n+1)^{2} \sqrt{\rho_{R} \rho_{B} \mu_{R} \mu_{B}}}{\sqrt{2}\left[n \rho_{R}+(n+1) \rho_{B}\right]\left(\sqrt{\rho_{R} \mu_{R}}+\sqrt{\rho_{B} \mu_{B}}\right)} .
$$

As Refs. [31, 34, 36], the second mode of the oscillation is considered in this work, i.e., $n=2$. The simulations are conducted in a $150 \times 300$ lattice domain with the boundary conditions the same as in the static droplet test. The densities of red and blue fluids are both fixed at $\rho_{c}=1$. Following Liang et al. [36], the interface profile is initialized as

$$
\rho^{N}(r, z)=\tanh \left(R_{D} \frac{1-\sqrt{\left(r-r_{c}\right)^{2} / R_{r}^{2}+\left(z-z_{c}\right)^{2} / R_{z}^{2}}}{\xi}\right),
$$

where $\left(r_{c}, z_{c}\right)=(0,150)$ is the center position of the ellipsoidal droplet; $R_{r}$ and $R_{z}$ are the half-axis lengths of the ellipsoid in the $r$ and $z$ directions, respectively; and the equilibrium radius $R_{D}$ is calculated by $R_{D}=\left(R_{r}^{2} R_{z}\right)^{1 / 3}$ (based on mass conservation of the droplet). With the initial interface profile, i.e. Eq.(41), one can determine the initial density fields by $\rho_{R}=\rho_{c}\left(1+\rho^{N}\right) / 2$ and $\rho_{B}=$ $\rho_{c}\left(1-\rho^{N}\right) / 2$. Fig.5 shows the snapshots of an oscillating droplet at different times for $R_{r}=40, R_{z}=55, \mu_{R}=2 \times 10^{-2}, \mu_{B}=2 \times 10^{-3}$, and $\sigma=5 \times 10^{-3}$. It is observed that the droplet oscillates with time until finally reaching an equilibrium spherical shape. The droplet oscillation is quantified by measuring the half-axis length $R_{r}$ as a function of time, which is plotted in Fig.6 for four different values of $\sigma$, i.e., $\sigma=2 \times 10^{-2}, 10^{-2}, 5 \times 10^{-3}$, and $10^{-3}$. Note in these cases that all the parameters are kept the same as those used in Fig.5 except $\sigma$. We can clearly see that $R_{r}$ fluctuates around $R_{D}$ during the droplet oscillation for all values of $\sigma$, but its maximum value decreases with decreasing $\sigma$. Besides, the oscillation period decreases as the interfacial tension is increased. Specifically, the oscillation periods $T_{L B M}$ computed by the present LBM are 6449, 9114.3, 13063.2 and 30046.8, respectively, for $\sigma=2 \times 10^{-2}, 10^{-2}, 5 \times 10^{-3}$ and $10^{-3}$, and their corresponding analytical solutions $T_{\text {anal }}$ are $6266.6,8929.1$, 12739.7, and 29260, where $T_{\text {anal }}=2 \pi / \omega_{2}$ and $\omega_{2}$ is given by Eq.(38). Evidently, the numerical predictions $T_{L B M}$ agree well with the analytical solutions $T_{a n a l}$, with a maximum relative error of around $2.9 \%$. 


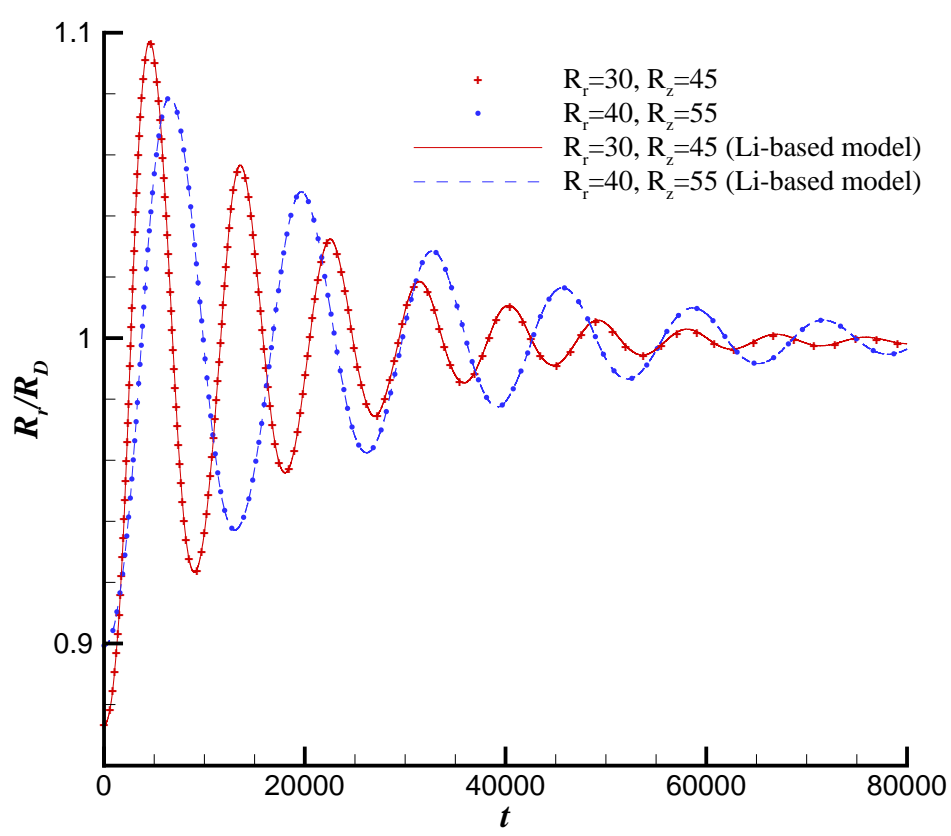

Figure 7: (Color Online) Time evolution of the half-axis length $R_{r}$ (normalized by the equilibrium radius $R_{D}$ ) at different initial sizes of the droplet for $\mu_{R}=2 \times 10^{-2}, \mu_{B}=2 \times 10^{-3}$, and $\sigma=5 \times 10^{-3}$. Note that the discrete symbols represent the simulation results from the color-gradient LBM proposed in Sect.2, while the lines represent the simulation results from the Li-based model presented in Appendix B. 


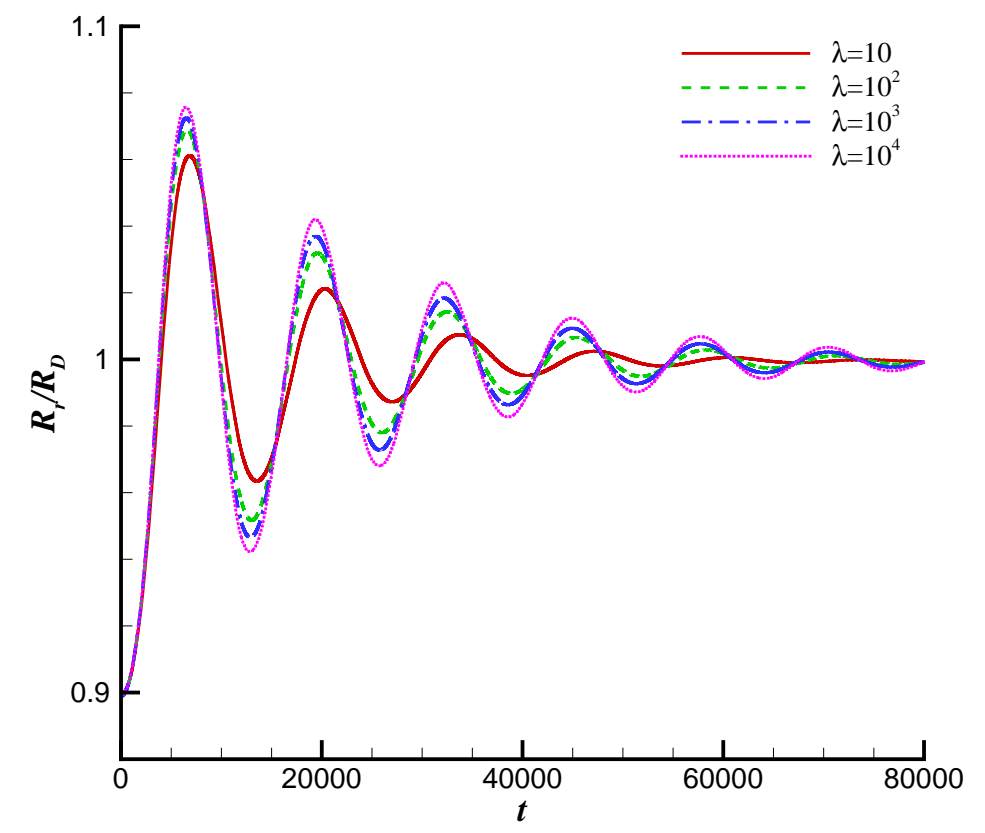

Figure 8: (Color Online) Time evolution of the half-axis length $R_{r}$ at different values of $\lambda$ for $R_{r}=40, R_{z}=55, \mu_{R}=0.05$, and $\sigma=5 \times 10^{-3}$. Note that the half-axis length $R_{r}$ is normalized by the equilibrium radius $R_{D}$.

Next, we examine the influence of the droplet size on the oscillation period. Fig.7 shows the temporal evolution of the half-axis length $R_{r}$ at $\mu_{R}=2 \times 10^{-2}$, $\mu_{B}=2 \times 10^{-3}$, and $\sigma=5 \times 10^{-3}$ for two different initial sizes of the droplet: $R_{r}=30, R_{z}=45$ and $R_{r}=40, R_{z}=55$. It is seen that decreasing the droplet size decreases the oscillation period. The computed oscillation periods for the large and small droplets are respectively 13063.2 and 9003.2, which are very close to their corresponding analytical values 12739.7 and 8674.5 , with the relative errors within $3.8 \%$.

Then, we further examine the method's capability for simulating the binary fluids with high viscosity ratio. Four different viscosity ratios are considered: $\lambda=10,10^{2}, 10^{3}$ and $10^{4}$, and they are achieved by adjusting $\mu_{B}$ while keeping 


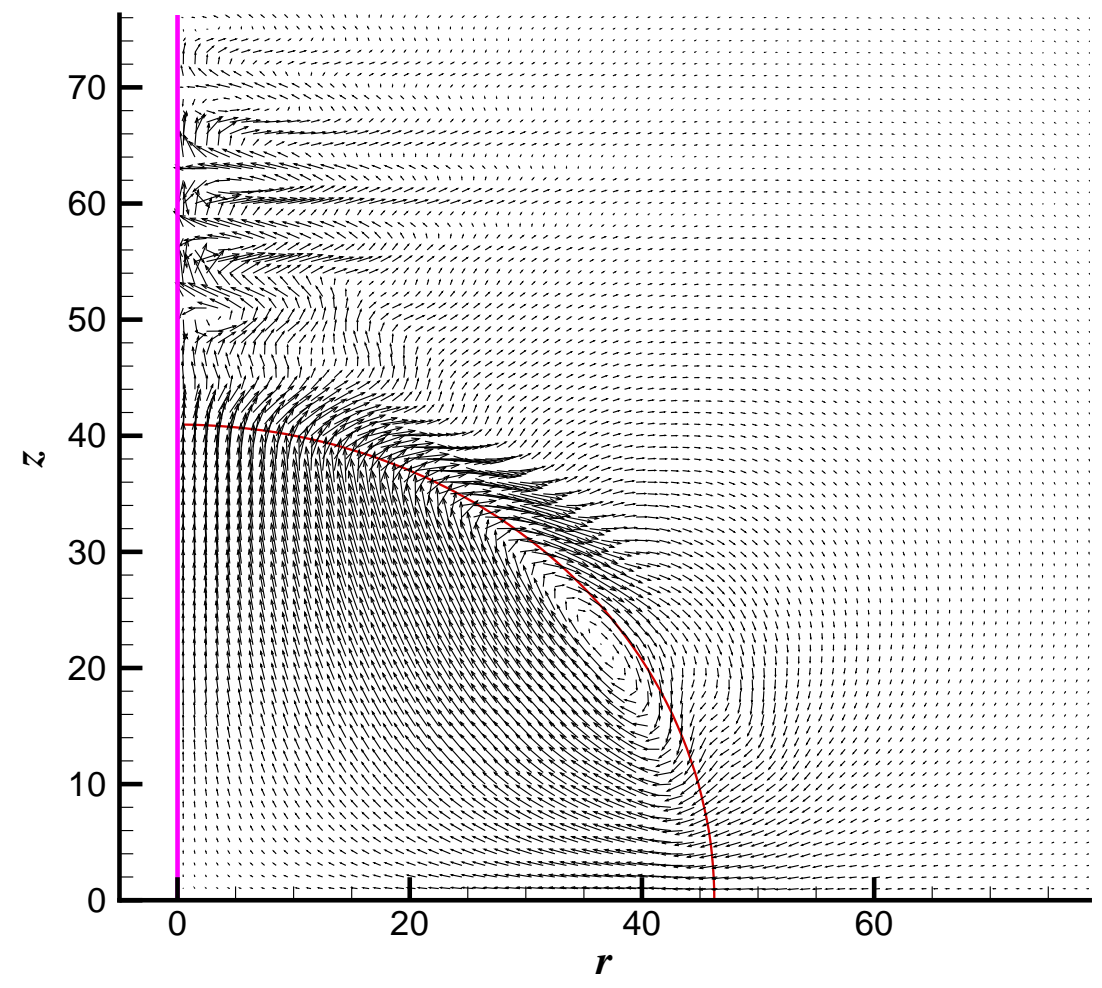

Figure 9: (Color Online) Velocity field at $t=20000$ timesteps for $R_{r}=40, R_{z}=55$, $\mu_{R}=0.05, \lambda=10^{4}$, and $\sigma=5 \times 10^{-3}$. Note that the velocity vectors are obtained by using the MRT model of Pooley et al. [48], and only a part of computational domain is illustrated in order to clearly show the local abnormal velocities. The red lines represent the droplet interface, while the pink line is the axis of symmetry $(r=0)$. 
$\mu_{R}$ fixed at 0.05 . Other parameters are given as follows: $R_{r}=40, R_{z}=55$, and $\sigma=5 \times 10^{-3}$. Fig.8 illustrates the temporal evolution of the half-axis length $R_{r}$ at various viscosity ratios. We can see that the simulations are stable for all the viscosity ratios considered, and the amplitude of oscillation (represented by $R_{r}$ ) increases with $\lambda$ since at larger $\lambda$ (which corresponds to smaller $\mu_{B}$ ), the viscous damping effect is reduced. In addition, as the viscosity ratio is increased the oscillation period decreases, but the decrease is insignificant for $\lambda \geq 10^{2}$. This can be more clearly seen in Table 2 , which shows the comparison between $T_{L B M}$ and $T_{\text {anal }}$ at various viscosity ratios. Overall, the computed oscillation periods are in agreement with the analytical results for all viscosity ratios, with a maximum relative error of around $6.2 \%$. Also, the relative error increases with increasing viscosity ratio, indicating that the present axisymmetric LBM has a lower predictive accuracy (although still acceptable) at higher viscosity ratio. It is interesting to make a remark concerning the choice of free parameters $s_{1}, s_{2}$ and $s_{4}$ in the diagonal relaxation matrix $\mathbf{S}$. These free parameters are not uniquely determined although Lallemand and Luo [64] provided some guidelines to choose some of them. It is therefore not surprising that a number of forms of diagonal relaxation matrix have been reported in literature with different free parameters, e.g., Refs. [73, 74, 63, 75, 62, 48, 76]. To date, a trialand-error approach is still required to find the most stable and reliable set of free parameters for a specific problem. For the simulations of droplet oscillation, we have found that the present MRT model with the choice of $s_{1}=1.63$, $s_{2}=1.14$, and $s_{4}=1.92$ produces more stable results than the well-known Two-Relaxation-Time (TRT) algorithm [73] $\left(s_{1}=s_{2}=\frac{1}{\tau}, s_{4}=\frac{8\left(2-s_{1}\right)}{8-s_{1}}\right)$, the MRT of Pooley et al. [48] $\left(s_{1}=s_{2}=s_{4}=1\right)$, and the MRT of Fakhari and Lee [76] $\left(s_{1}=s_{2}=1, s_{4}=1.7\right)$. For example, in the case of $\lambda=10^{4}$, the simulation is stable at all times for the present MRT model, but diverges at approximately 19000 timesteps for the TRT, 230000 timesteps for the MRT of Pooley et al. and 92000 timesteps for the MRT of Fakhari and Lee, respectively. Note that, although the simulation does not blow up until $t=230000$ when the MRT of Pooley et al. is used, some abnormal velocities have been 
clearly observed near the axis of symmetry and the droplet interface inside the blue (less viscous) fluid at an earlier time i.e. $t=20000$ (see Fig.9). The magnitude of abnormal velocities increases gradually with time, eventually leading to divergence of the simulation. In addition, the MRT model should be more stable than the BGK model even if the choice of free parameters is not optimal $[64,73,62,63,50]$. This is demonstrated by our simulations, in which the BGK model breaks down at $t=4000$, much earlier than all of the MRT models we have tested (note that the model will be more unstable if it breaks down earlier). Based on the limited numerical comparisons shown here, the guidelines given by Lallemand and Luo [64] (recall that our free parameters are chosen following their recommendation) seem to be applicable as well in the present color-gradient MRT model. However, further study is required to provide more insights in determining optimal parameters.

Table 2: Comparison between the computed $\left(T_{L B M}\right)$ and analytical $\left(T_{\text {anal }}\right)$ oscillation periods at various viscosity ratios for $R_{r}=40, R_{z}=55, \sigma=5 \times 10^{-3}$, and $\mu_{R}=0.05$.

\begin{tabular}{c|cccc}
\hline \hline$\lambda$ & 10 & $10^{2}$ & $10^{3}$ & $10^{4}$ \\
\hline$T_{L B M}$ & 13448.1 & 13008.9 & 12890.0 & 12824.0 \\
$T_{\text {anal }}$ & 13144.8 & 12456.2 & 12174.8 & 12077.7 \\
$E_{r}=\frac{\left|T_{L B M}-T_{\text {anal }}\right|}{\left|T_{\text {anal }}\right|} \times 100 \%$ & $2.31 \%$ & $4.44 \%$ & $5.87 \%$ & $6.19 \%$ \\
\hline
\end{tabular}

In addition to the MRT model, which can enhance the stability for simulation of multiphase/multicomponent flows with high viscosity ratio, we also implement a variant of the BGK model, which is based on a regularization of the pre-collision PDFs. This model is known as the RLB model, which was proposed independently by Latt and Chopard [77], Chen et al. [78], and Zhang et al. [79]. It was found that, in comparison with the BGK model, the RLB model offers an improvement in both stability and accuracy without adding any substantial complication. Later, the RLB model was also demonstrated to perform very well in the multicomponent interparticle-potential LBM [80]. In view of the advantages of RLB model, it is worthwhile to extend it to the present 
color-gradient LBM and test its effectiveness in the simulation of axisymmetric multicomponent flows with high viscosity ratio. Inspired by the previous work$\mathrm{s}[77,81,82]$, we derive a regularized expression for computing the incoming PDFs before collision, which is given by

$$
f_{i}^{k} \approx f_{i}^{k, e q}+f_{i}^{k,(1)}=f_{i}^{k, e q}+\frac{w_{i}}{2 c_{s}^{4}} Q_{i \alpha \beta} \Pi_{\alpha \beta}^{k, n e q}-\frac{w_{i}}{2 c_{s}^{2}} e_{i \alpha} f_{s \alpha}^{k},
$$

where $Q_{i \alpha \beta}=e_{i \alpha} e_{i \beta}-c_{s}^{2} \delta_{\alpha \beta}, f_{s \alpha}^{k}=A_{k} f_{s \alpha}$, and $\Pi_{\alpha \beta}^{k, n e q}=\sum_{i}\left(f_{i}^{k}-f_{i}^{k, e q}\right) e_{i \alpha} e_{i \beta}$ is the nonequilibrium component of the momentum flux tensor. It can be easily proved that $f_{i}^{k,(1)}$ in Eq.(42) satisfies the following constraints:

$$
\begin{aligned}
\sum_{i} f_{i}^{k,(1)} & =0 \\
\sum_{i} f_{i}^{k,(1)} e_{i \alpha} & =-\frac{1}{2} f_{s \alpha}^{k}, \\
\sum_{i} f_{i}^{k,(1)} e_{i \alpha} e_{i \beta} & =-\rho_{k} c_{s}^{2} \tau\left(\partial_{\beta} u_{\alpha}+\partial_{\alpha} u_{\beta}\right)-\frac{1}{2}\left(u_{\alpha} f_{s \beta}^{k}+u_{\beta} f_{s \alpha}^{k}\right) .
\end{aligned}
$$

Except for an additional, straightforward regularization step as shown in Eq.(42), all the constituents of the collision operator are kept the same as those in the color-gradient BGK model, i.e. Eqs.(6), (20) and (22). We finally test the colorgradient RLB model's capability through the simulation of droplet oscillation with $\lambda=10^{4}$. All the parameters and boundary conditions are identical to those used in Fig.9. It is found that the RLB model produces stable results at all times, and the obtained results are in excellent agreement with the results of our MRT model that uses the free parameters recommended by Lallemand and Luo [64] (see Fig.10). This suggests that the color-gradient RLB model is effective for the quantitative study of axisymmetric multicomponent flows, and is numerically accurate and stable even for very small relaxation time $\tau$ and high viscosity ratio, which remain a challenge for many of the MRT models.

\subsection{Breakup of a liquid thread}

In order to reveal the capability of the present LBM in the simulation of large topological changes, we consider the problem of the breakup of a liquid 


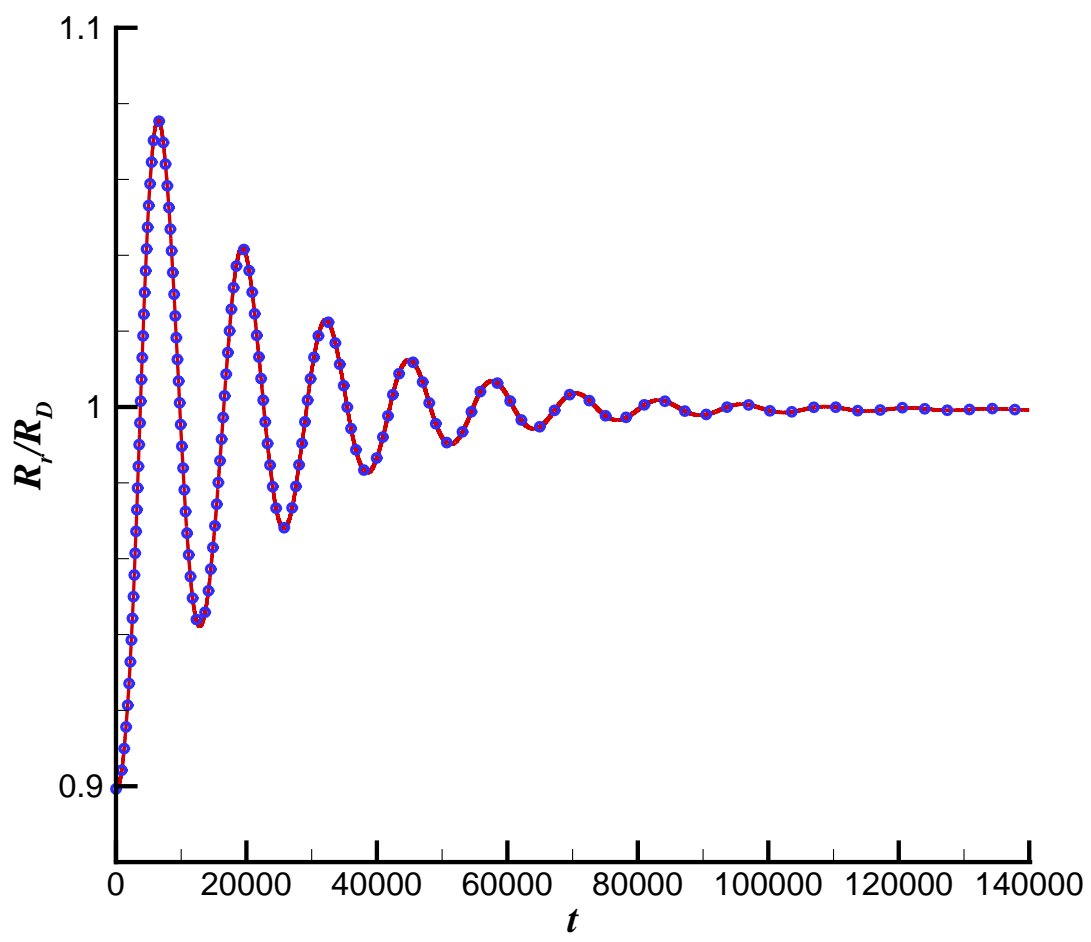

Figure 10: (Color Online) Time evolution of the half-axis length $R_{r}$ (normalized by the equilibrium radius $R_{D}$ ) for $R_{r}=40, R_{z}=55, \mu_{R}=0.05, \lambda=10^{4}$, and $\sigma=5 \times 10^{-3}$. Note that the discrete symbols represent the simulation results from the color-gradient RLB model, while the lines represent the simulation results from the color-gradient LBM proposed in Sect.2. 
thread into multiple droplets. This problem was first studied experimentally by Plateau [83] and later investigated theoretically by Rayleigh [84]. Through a linear stability analysis, Rayleigh [84] showed that a cylindrical liquid thread of radius $R_{c}$ is unstable if the wavelength $\lambda_{d}$ of a disturbance is greater than its circumference $2 \pi R_{c}$. In other words, the liquid thread is unstable when the wave number $k^{*}\left(k^{*}=2 \pi R_{c} / \lambda_{d}\right)$ is less than unity, and stable when $k^{*}$ is larger than unity. Several preliminary numerical tests conducted with $k^{*}>1$ do show that the liquid thread does not break up, consistent with the linear stability theory. In the following simulations, we will focus on the cases with breakup (i.e., $k^{*}<1$ ), and compare the simulated results with some available literature data.

The simulations are run in a $300 \times \lambda_{d}$ lattice domain with the same boundary conditions as in the static and oscillating droplet tests. A disturbance is introduced by specifying the initial interface profile as

$$
\rho^{N}(r, z)=\tanh \frac{R_{c}+d-r}{\xi},
$$

where $d$ is the interfacial disturbance imposed on the liquid thread and is given by $d=0.1 R_{c} \cos \left(2 \pi z / \lambda_{d}\right)$. In the absence of disturbance, Eq.(44) represents a perfect cylindrical column with radius $R_{c}$, which is taken as 60 in our study. Note that the disturbance can be also introduced in other forms, e.g., a fluctuation on the initial velocity field in Ref. [35]. Seven cases with the wavelengths $\lambda=\{420,500,600,800,1000,1200,1800\}$ are considered, which correspond to seven different wave numbers, i.e. $k^{*}=\{0.90,0.75,0.63,0.47,0.38,0.31,0.21\}$. The densities of both fluids are set to be unity, and the interfacial tension $\sigma$ is $6 \times 10^{-3}$. The Ohnesorge number, which is defined by $O h=\mu_{R} / \sqrt{\rho_{R} \sigma R_{c}}$, is kept at 0.1 . Using the aforementioned parameters, one can easily get $\mu_{R}=0.06$, and $\mu_{B}$ is then determined by the viscosity ratio $\lambda$, which is chosen as 10 unless otherwise stated.

Fig.11 shows the snapshots of the breakup of a liquid thread for three typical wave numbers, where time is normalized by the capillary time, $t_{c a p}=\sqrt{R_{c}^{3} \rho_{R} / \sigma}$. It is observed that the interfacial disturbances gradually grow with time for all 


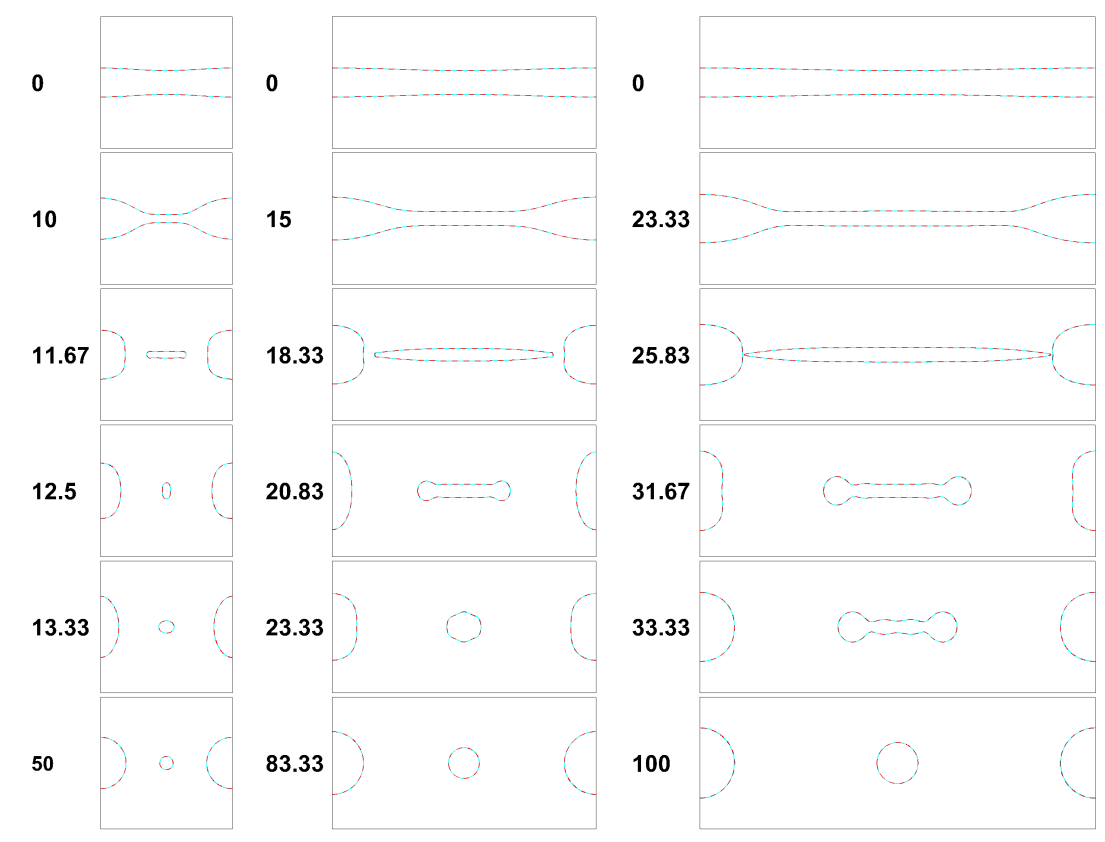

Figure 11: (Color Online) Snapshots of the breakup of a liquid thread at $\lambda=10$ for different wave numbers, left panel: $k^{*}=0.63$, middle panel: $k^{*}=0.31$, right panel: $k^{*}=0.21$. Time is normalized by the capillary time $\sqrt{R_{c}^{3} \rho_{R} / \sigma}$. Note that the cyan solid lines represent the simulation results from the color-gradient LBM proposed in Sect.2, while the red dashed lines represent the simulation results from the Li-based model presented in Appendix B. 


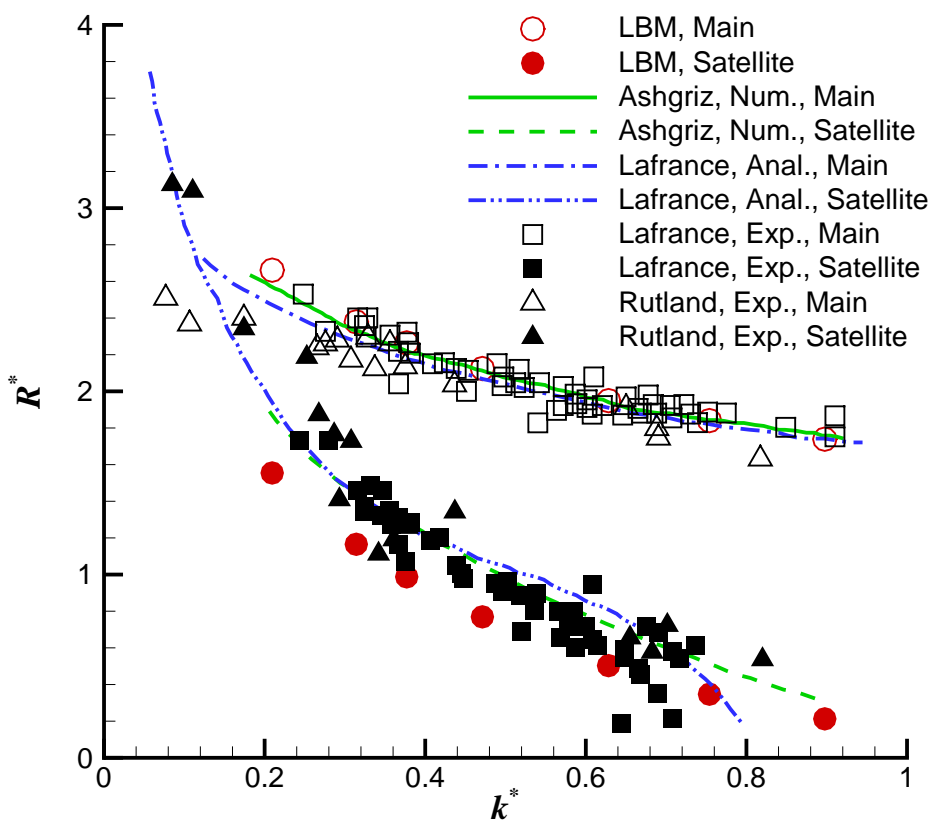

Figure 12: (Color Online) $R^{*}$ as a function of the wave number for $\lambda=10 . R^{*}$ is the droplet radius normalized by $R_{c}$. 
of the wave numbers. Then the liquid thread in the middle region progressively thins and, at the same time the ends enlarge until the thread breaks up, forming a thin ligament as well as a main droplet (see, for example, time 25.83 at $k^{*}=$ 0.21). Afterwards, the ligament contracts continuously due to the dominant interfacial tension, eventually leading to a spherical satellite droplet formed. During the contraction of the liquid ligament, a pair of droplets can be found at the end of the ligament, and they exhibit an increasing trend to pinch-off from the middle portion as the wave number increases. Therefore, it is expected that a liquid ligament can break up into multiple droplets as long as it is sufficiently long, consistent with the previous findings $[85,86,36]$. In addition, we also quantify the sizes of the main and satellite droplets at various wave numbers, which are plotted in Fig.12. For the purpose of comparison, previous results including the finite element results by Ashgriz and Mashayek [85], the analytical solutions and experimental data of Lafrance [87], as well as the experimental data of Rutland and Jameson [88] are also presented. Evidently, the main and satellite droplets both decrease in size with increasing the wave number; and also, our simulation results show good agreement with available literature data in general, providing further validation of the present axisymmetric colorgradient LBM.

To show the influence of the viscosity ratio on the breakup of the liquid thread, we also simulate the case of $\lambda=10^{2}$, which is achieved by varying $\mu_{B}$ while keeping all the other parameters the same as used in the case of $\lambda=10$. Fig.13 depicts the snapshots of the breakup of a liquid thread at $\lambda=10^{2}$ for $k^{*}=0.63$ (left), 0.31 (middle), and 0.21 (right), where the snapshots are taken at the times exactly the same as in the case of $\lambda=10$. By comparing the cases of $\lambda=10$ and $\lambda=10^{2}$, we can find that the viscosity ratio can affect not only the interface structure but also its dynamical evolution. For example, the liquid thread breaks up earlier at $\lambda=10^{2}$ for each of the wave numbers, suggesting that increasing viscosity ratio can speed up the growth of Rayleigh instability. However, it is found that the viscosity ratio has a negligible effect on the sizes of the main and satellite droplets formed. This could explain why the data 


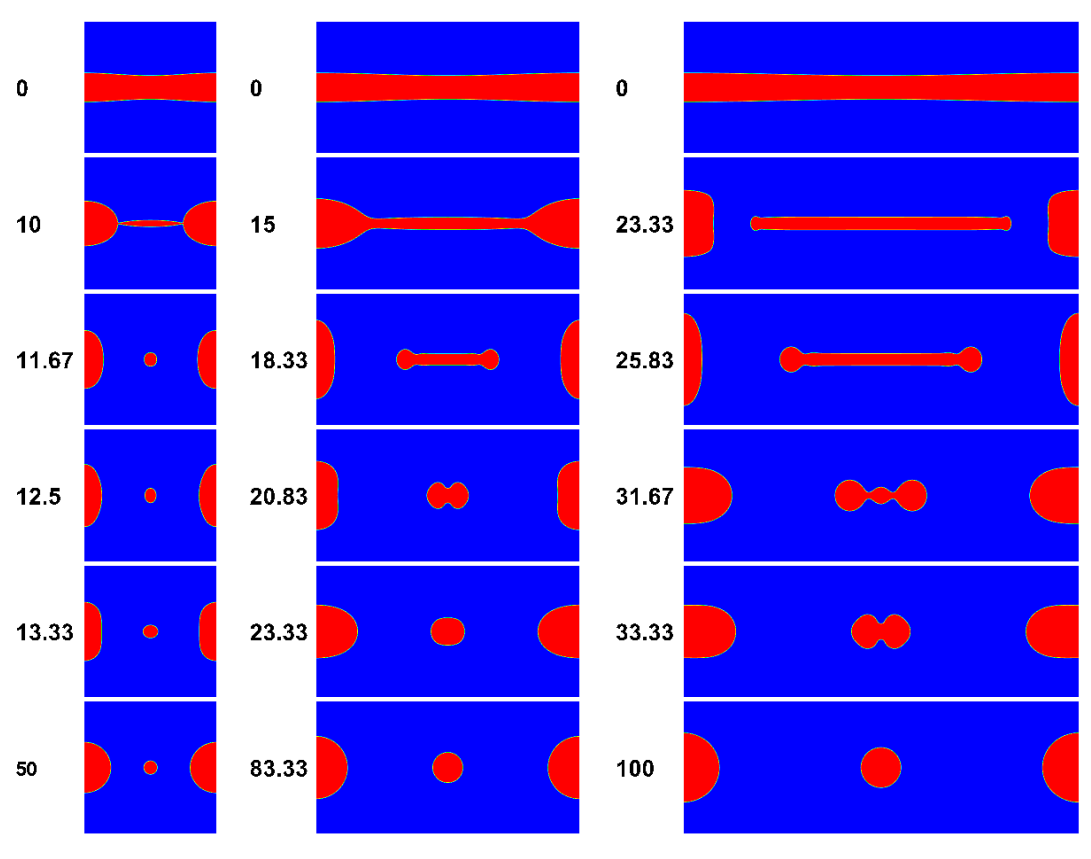

Figure 13: (Color Online) Snapshots of the breakup of a liquid thread at $\lambda=10^{2}$ for different wave numbers, left panel: $k^{*}=0.63$, middle panel: $k^{*}=0.31$, right panel: $k^{*}=0.21$. Time is normalized by the capillary time $\sqrt{R_{c}^{3} \rho_{R} / \sigma}$. 
from different literature sources are all comparable with those experimentally obtained by Rutland and Jameson [88] in the $k^{*}-R^{*}$ diagram (where $R^{*}$ is the droplet radius normalized by $R_{c}$ ), although they have considered different viscosity ratios.

\section{Conclusions}

A color-gradient LBM is developed for simulating axisymmetric multicomponent flows for a broad range of viscosity ratios. Like the Cartesian colorgradient models, this method uses a collision operator that is a combination of three separate parts, namely the single-component collision operator, perturbation operator, and the recoloring operator. In order to recover correctly the continuity and momentum equations in each single-component region, a source term is added into the single-component collision operator to account for the axisymmetric contributions. In the perturbation step, an interfacial force of axisymmetric form is derived using the CSF concept together with a coordinate transformation, and is incorporated into the LBM through the body force model of Guo et al. [61]. A recoloring operator proposed by Latva-Kokko and Rothman [47] is extended to the axisymmetric case for producing the phase segregation and guaranteeing the immiscibility of both fluids. To improve the numerical stability for solving binary fluids with high viscosity ratio, the axisymmetric color-gradient LBM is implemented in the MRT framework instead of the standard BGK approximation. The usefulness of accuracy of the method are assessed by several typical numerical examples, including static droplet test, oscillation of a viscous droplet, and breakup of a liquid thread. The former two examples show that the present LBM is able to accurately capture the interface, produce low unphysical spurious velocities, and simulate the viscosity ratios up to $10^{4}$ with acceptable numerical accuracy. In the last example, the detailed comparison between the computed results and the previous literature data shows that the present LBM can provide reasonable predictions of the thread breakup caused by the Rayleigh instability. In addition, the viscosity ratio is found to 
significantly affect the growth of the Rayleigh instability, but it has a negligible effect on the sizes the formed droplets. In our future work, we would like to apply the present color-gradient LBM for more sophisticated problems, e.g. diesel spray formation and breakup, and compare our simulation results with those reported by Falcucci et al. [89].

Appendix A. Derivation of axisymmetric Navier-Stokes equations using the Chapman-Enskog expansion

In the absence of the perturbation and recoloring operators, the collision operator in Eq. (2) can be simplified as $\Omega_{i}^{k}=\left(\Omega_{i}^{k}\right)^{(1)}$, and one can rewrite Eq. (1) as

$f_{i}^{k}\left(\vec{x}+\vec{e}_{i} \delta_{t}, t+\delta_{t}\right)=f_{i}^{k}(\vec{x}, t)-\frac{f_{i}^{k}(\vec{x}, t)-f_{i}^{k, e q}(\vec{x}, t)}{\tau}+\delta_{t} h_{i}^{k}\left(\vec{x}+\vec{e}_{i} \delta_{t} / 2, t+\delta_{t} / 2\right)$,

with $k=R$ or $B$ and $f_{i}^{k, e q}$ given by Eq. (7). Introducing the Chapman-Enskog expansion,

$$
\begin{aligned}
f_{i}^{k}\left(\vec{x}+\vec{e}_{i} \delta_{t}, t+\delta_{t}\right) & =\sum_{n=0}^{\infty} \frac{\epsilon^{n}}{n !} D_{t}^{n} f_{i}^{k}(\vec{x}, t), \\
f_{i}^{k} & =\sum_{n=0}^{\infty} \epsilon^{n} f_{i}^{k,(n)}, \\
h_{i}^{k}\left(\vec{x}+\vec{e}_{i} \delta_{t} / 2, t+\delta_{t} / 2\right) & =\sum_{n=0}^{\infty} \frac{(\epsilon / 2)^{n}}{n !} D_{t}^{n} h_{i}^{k}(\vec{x}, t),
\end{aligned}
$$

where $\epsilon=\delta_{t}$ and $D_{t} \equiv\left(\partial_{t}+\mathbf{e}_{i} \cdot \nabla\right)$, the following equations are obtained up to second order in the parameter $\epsilon$ :

$$
\begin{array}{ll}
\mathcal{O}\left(\epsilon^{0}\right): & f_{i}^{k,(0)}=f_{i}^{k, e q} \\
\mathcal{O}\left(\epsilon^{1}\right): & D_{t} f_{i}^{k,(0)}=-\frac{1}{\tau} f_{i}^{k,(1)}+h_{i}^{k}, \\
\mathcal{O}\left(\epsilon^{2}\right): & D_{t} f_{i}^{k,(1)}+\frac{1}{2} D_{t}^{2} f_{i}^{k,(0)}=-\frac{1}{\tau} f_{i}^{k,(2)}+\frac{1}{2} D_{t} h_{i}^{k} .
\end{array}
$$

Using Eq.(A.6), Eq.(A.7) can be written as

$$
\left(1-\frac{1}{2 \tau}\right) D_{t} f_{i}^{k,(1)}=-\frac{1}{\tau} f_{i}^{k,(2)} .
$$


Note that one can use the following solvability conditions for $f_{i}^{k,(n)}(n=1,2, \cdots)$,

$$
\sum_{i} f_{i}^{k,(n)}=0, \quad \sum_{k} \sum_{i} f_{i}^{k,(n)} e_{i \alpha}=0
$$

and the conditions for $f_{i}^{k, e q}$ and $h_{i}^{k}$ are

$$
\begin{aligned}
& \sum_{i} f_{i}^{k, e q}=\rho_{k}, \quad \sum_{i} f_{i}^{k, e q} e_{i \alpha}=\rho_{k} u_{\alpha}, \\
& \sum_{i} f_{i}^{k, e q} e_{i \alpha} e_{i \beta}=\rho_{k} u_{\alpha} u_{\beta}+\rho_{k} c_{s}^{2} \delta_{\alpha \beta}, \\
& \sum_{i} f_{i}^{k, e q} e_{i \alpha} e_{i \beta} e_{i \gamma}=\rho_{k} c_{s}^{2}\left(u_{\alpha} \delta_{\beta \gamma}+u_{\beta} \delta_{\alpha \gamma}+u_{\gamma} \delta_{\alpha \beta}\right), \\
& \sum_{i} h_{i}^{k} e_{i \alpha}=H_{\alpha}^{k}, \quad \sum_{i} h_{i}^{k} e_{i \alpha} e_{i \beta}=-\frac{\rho_{k} u_{r}}{r} c_{s}^{2} \delta_{\alpha \beta},
\end{aligned}
$$

where $H_{\alpha}^{k}$ is defined by Eq. (11).

From Eq.(A.6) + Eq.(A.8) $\times \epsilon$, one has

$$
D_{t} f_{i}^{k,(0)}+\epsilon\left(1-\frac{1}{2 \tau}\right) D_{t} f_{i}^{k,(1)}=-\frac{1}{\tau}\left(f_{i}^{k,(1)}+f_{i}^{k,(2)}\right)+h_{i}^{k} .
$$

Summation of the above equation over $i$ and $k$ provides

$$
\partial_{t} \rho+\partial_{\alpha}\left(\rho u_{\alpha}\right)=-\frac{\rho u_{r}}{r} .
$$

In the incompressible limit, the density of the fluid mixture is assumed to be small enough, and Eq.(A.12) can lead to the continuity equation (3).

Taking $\sum_{k} \sum_{i}[$ Eq.(A.6) + Eq.(A.8) $\times \epsilon]$ yields

$$
\partial_{t}\left(\rho u_{\alpha}\right)+\partial_{\beta} \Pi_{\alpha \beta}^{(0)}=\partial_{\beta} \Gamma_{\alpha \beta}^{(1)}+\sum_{k} H_{\alpha}^{k}
$$

where

$$
\Pi_{\alpha \beta}^{(0)}=\sum_{k} \sum_{i} f_{i}^{k,(0)} e_{i \alpha} e_{i \beta}=\rho c_{s}^{2} \delta_{\alpha \beta}+\rho u_{\alpha} u_{\beta}
$$

and

$$
\Gamma_{\alpha \beta}^{(1)}=-\epsilon\left(1-\frac{1}{2 \tau}\right) \sum_{k} \sum_{i} f_{i}^{k,(1)} e_{i \alpha} e_{i \beta} .
$$

Substitution of Eq.(11) into Eq.(A.13) results in

$$
\partial_{t}\left(\rho u_{\alpha}\right)+\partial_{\beta}\left(\rho u_{\alpha} u_{\beta}\right)=-\partial_{\alpha}\left(\rho c_{s}^{2}\right)+\partial_{\beta} \Gamma_{\alpha \beta}^{(1)}+\frac{\mu\left(\partial_{r} u_{\alpha}+\partial_{\alpha} u_{r}\right)}{r}-\frac{2 \mu u_{r}}{r^{2}} \delta_{\alpha r}-\frac{\rho u_{\alpha} u_{r}}{r} .
$$


Applying Eq.(A.6) one can rewrite Eq.(A.15) as

$$
\begin{aligned}
& \Gamma_{\alpha \beta}^{(1)}=\epsilon\left(\tau-\frac{1}{2}\right) \sum_{k} \sum_{i} D_{t} f_{i}^{k,(0)} e_{i \alpha} e_{i \beta}-\epsilon\left(\tau-\frac{1}{2}\right) \sum_{k} \sum_{i} h_{i}^{k} e_{i \alpha} e_{i \beta} \\
= & \epsilon\left(\tau-\frac{1}{2}\right)\left(\partial_{t} \Pi_{\alpha \beta}^{(0)}+\partial_{\gamma} \sum_{k} \sum_{i} f_{i}^{k,(0)} e_{i \alpha} e_{i \beta} e_{i \gamma}\right)+\epsilon\left(\tau-\frac{1}{2}\right) \frac{\rho u_{r}}{r} c_{s}^{2} \delta_{\alpha \beta} \\
= & \epsilon\left(\tau-\frac{1}{2}\right)\left[\partial_{t} \Pi_{\alpha \beta}^{(0)}+c_{s}^{2} \partial_{\gamma}\left(\rho u_{\alpha} \delta_{\beta \gamma}+\rho u_{\beta} \delta_{\alpha \gamma}+\rho u_{\gamma} \delta_{\alpha \beta}\right)+\frac{\rho u_{r}}{r} c_{s}^{2} \delta_{\alpha \beta}\right](\mathrm{A} .1
\end{aligned}
$$

Through the dimensional analysis, one can obtain that the ratio of the first to the second terms in the square bracket of Eq.(A.17) has the order

$$
\mathcal{O}\left(\frac{\partial_{t} \Pi_{\alpha \beta}^{(0)}}{c_{s}^{2} \partial_{\gamma}\left(\rho u_{\alpha} \delta_{\beta \gamma}+\rho u_{\beta} \delta_{\alpha \gamma}+\rho u_{\gamma} \delta_{\alpha \beta}\right)}\right)=\mathcal{O}\left(M a^{2}\right),
$$

where $M a$ is the Mach number. It shows that the term $\partial_{t} \Pi_{\alpha \beta}^{(0)}$ is very small compared with $c_{s}^{2} \partial_{\gamma}\left(\rho u_{\alpha} \delta_{\beta \gamma}+\rho u_{\beta} \delta_{\alpha \gamma}+\rho u_{\gamma} \delta_{\alpha \beta}\right)$ and can be neglected if $M a \ll$ 1 [54]; hence Eq.(A.17) becomes

$$
\begin{aligned}
\Gamma_{\alpha \beta}^{(1)} & =\epsilon\left(\tau-\frac{1}{2}\right)\left[c_{s}^{2} \partial_{\gamma}\left(\rho u_{\alpha} \delta_{\beta \gamma}+\rho u_{\beta} \delta_{\alpha \gamma}+\rho u_{\gamma} \delta_{\alpha \beta}\right)+\frac{\rho u_{r}}{r} c_{s}^{2} \delta_{\alpha \beta}\right] \\
& =\epsilon\left(\tau-\frac{1}{2}\right) c_{s}^{2}\left[\rho\left(\partial_{\beta} u_{\alpha}+\partial_{\alpha} u_{\beta}\right)+u_{\alpha} \partial_{\beta} \rho+u_{\beta} \partial_{\alpha} \rho+u_{\gamma} \partial_{\gamma} \rho \delta_{\alpha \beta}\right] \\
& =\epsilon\left(\tau-\frac{1}{2}\right) c_{s}^{2} \rho\left(\partial_{\beta} u_{\alpha}+\partial_{\alpha} u_{\beta}\right),
\end{aligned}
$$

in which we have used the continuity equation and neglected the terms of $\mathcal{O}\left(M a^{3}\right)$. Substitution of Eq.(A.19) into Eq.(A.16) leads to

$$
\begin{aligned}
\partial_{t}\left(\rho u_{\alpha}\right)+\partial_{\beta}\left(\rho u_{\alpha} u_{\beta}\right)= & -\partial_{\alpha}\left(\rho c_{s}^{2}\right)+\partial_{\beta}\left[\left(\tau-\frac{1}{2}\right) c_{s}^{2} \rho \delta_{t}\left(\partial_{\beta} u_{\alpha}+\partial_{\alpha} u_{\beta}\right)\right] \\
& +\frac{\mu\left(\partial_{r} u_{\alpha}+\partial_{\alpha} u_{r}\right)}{r}-\frac{2 \mu u_{r}}{r^{2}} \delta_{\alpha r}-\frac{\rho u_{\alpha} u_{r}}{r},
\end{aligned}
$$

which reduces to the exact momentum equation (5) in the incompressible limit if the pressure and the viscosity are given by Eq.(12) and Eq.(13), respectively.

\section{Appendix B. An accurate version of color-gradient model for axisym- metric multicomponent flows}

To test the accuracy of the LBM proposed in Sect.2, we also present an accurate version of the color-gradient model for axisymmetric multicomponent flows. 
In this model, the single-component collision operator and the perturbation operator are derived based on the axisymmetric single-phase LBM proposed by Li et al. [53], and the recoloring operator takes the same form as the one shown in Eq.(22). In view of its theoretical basis, this model is called as Li-based model here. The Li-based model can recover the correct continuity and momentum equations in the axisymmetric coordinate system to the second-order accuracy; and also, it does not need to introduce any approximations in the implementation. Thus, its numerical results are considered 'accurate' and can be regarded as benchmark solutions. Following Li et al. [53], the single-component collision operator and the perturbation operator are given by

$$
\begin{aligned}
\left(\Omega_{i}^{k}\right)^{(1)} & =-\frac{1}{\tau^{\prime}}\left[f_{i}^{k}(\vec{x}, t)-f_{i}^{k, e q}(\vec{x}, t)\right]+\left(1-\frac{1}{2 \tau^{\prime}}\right) \phi_{i}^{k}(\vec{x}, t), \\
\left(\Omega_{i}^{k}\right)^{(2)} & =A_{k}\left(1-\frac{1}{2 \tau^{\prime}}\right) \frac{\left(e_{i \alpha}-u_{\alpha}\right) f_{s \alpha}(\vec{x}, t)}{\rho_{k} c_{s}^{2}} f_{i}^{k, e q}(\vec{x}, t) \delta_{t},
\end{aligned}
$$

with

$$
\phi_{i}^{k}=\left[\frac{\left(e_{i \alpha}-u_{\alpha}\right)}{c_{s}^{2}}\left(-\frac{2 \nu u_{\alpha}}{r^{2}} \delta_{\alpha r}\right)-\frac{u_{r}}{r}\right] f_{i}^{k, e q} \delta_{t},
$$

where $\tau^{\prime}=(\tau+0.5) /\left[1+\tau \delta_{t}\left(e_{i \alpha} / r\right)\right]$.

The macroscopic variables can be calculated by

$$
\begin{aligned}
\rho_{k}(\vec{x}, t) & =\frac{\sum_{i} f_{i}^{k}(\vec{x}, t)}{\left(1+\frac{\delta_{t} u_{r}(\vec{x}, t)}{2 r}\right)}, \\
u_{\alpha}(\vec{x}, t) & =\frac{\sum_{k} \sum_{i} f_{i}^{k}(\vec{x}, t) e_{i \alpha}+0.5 f_{s \alpha}(\vec{x}, t) \delta_{t}}{\sum_{k} \sum_{i} f_{i}^{k}(\vec{x}, t)+\left(\delta_{t} \frac{\mu}{r^{2}}\right) \delta_{\alpha r}} .
\end{aligned}
$$

As seen from Eqs.(B.4) and (B.5), the densities and the fluid velocity are coupled in a nonlinear fashion. To avoid such a nonlinear coupling, we first compute $\rho^{N}$ by

$$
\rho^{N}(\vec{x}, t)=\frac{\rho^{R}(\vec{x}, t)-\rho^{B}(\vec{x}, t)}{\rho^{R}(\vec{x}, t)+\rho^{B}(\vec{x}, t)}=\frac{\sum_{i} f_{i}^{R}(\vec{x}, t)-\sum_{i} f_{i}^{B}(\vec{x}, t)}{\sum_{i} f_{i}^{R}(\vec{x}, t)+\sum_{i} f_{i}^{B}(\vec{x}, t)} .
$$

Once the value of $\rho^{N}$ is obtained, the interfacial force can be calculated by Eq.(18), and then the fluid velocity can be calculated by Eq.(B.5). Finally, the densities of red and blue fluids are obtained through Eq.(B.4).

Figs.2, 7 and 11 show the comparison between the simulation results obtained by the Li-based model and those obtained by the color-gradient LBM 
described in Sect.2. Excellent agreement is observed in all of these figures, indicating that the color-gradient LBM described in Sect.2 can provide 'accurate' prediction of the axisymmetric multicomponent flows albeit that the centered scheme with an explicit approximation is used for the axisymmetric contributions. In addition, the color-gradient LBM described in Sect.2 is as simple as the original color-gradient model in form (roughly the same except several small additives or variants), so it is preferred and is the focus of this work.

\section{Acknowledgements}

This work is financially supported by the Thousand Youth Talents Program for Distinguished Young Scholars, the National Natural Science Foundation of China (No. 51506168) and the China Postdoctoral Science Foundation (No. 2016M590943). This work is also supported by the UK's Engineering and Physical Sciences Research Council (EPSRC) under grant EP/L00030X/1.

[1] S. O. Unverdi, G. Tryggvason, A front-tracking method for viscous, incompressible, multi-fluid flows, Journal of Computational Physics 100 (1) (1992) 25-37.

[2] C. Hirt, B. Nichols, Volume of fluid (VOF) method for the dynamics of free boundaries, J. Comput. Phys. 39 (1981) 201-225.

[3] S. Osher, R. P. Fedkiw, Level sets methods and dynamic implicit surfaces, Springer, 2003.

[4] J. A. Sethian, P. Smereka, Level set methods for fluid interfaces, Ann. Rev. Fluid Mech. 35 (2003) 341-372.

[5] J. Zhang, Lattice Boltzmann method for microfluidics: models and applications, Microfluid. Nanofluid. 10 (1) (2011) 1-28.

[6] C. K. Aidun, J. R. Clausen, Lattice-Boltzmann method for complex flows, Annu. Rev. Fluid Mech. 42 (1) (2010) 439-472. 
[7] R. Benzi, S. Succi, M. Vergassola, The lattice Boltzmann equation: theory and applications, Physics Reports 222 (3) (1992) 145-197.

[8] S. Chen, G. D. Doolen, Lattice Boltzmann method for fluid flows, Annu. Rev. Fluid Mech. 30 (1) (1998) 329-364.

[9] A. K. Gunstensen, D. H. Rothman, S. Zaleski, G. Zanetti, Lattice Boltzmann model of immiscible fluids, Phys. Rev. A 43 (8) (1991) 4320-4327.

[10] I. Halliday, R. Law, C. M. Care, A. Hollis, Improved simulation of drop dynamics in a shear flow at low Reynolds and capillary number, Phys. Rev. E 73 (5) (2006) 056708.

[11] T. Reis, T. N. Phillips, Lattice Boltzmann model for simulating immiscible two-phase flows, J. Phys. A-Math. Theor. 40 (14) (2007) 4033-4053.

[12] H. Liu, A. J. Valocchi, Q. Kang, Three-dimensional lattice Boltzmann model for immiscible two-phase flow simulations, Phys. Rev. E 85 (2012) 046309.

[13] M. R. Swift, E. Orlandini, W. R. Osborn, J. M. Yeomans, Lattice Boltzmann simulations of liquid-gas and binary fluid systems, Phys. Rev. E 54 (5) (1996) 5041-5052.

[14] H. Zheng, C. Shu, Y. Chew, A lattice Boltzmann model for multiphase flows with large density ratio, J. Comput. Phys. 218 (1) (2006) 353-371.

[15] T. Lee, L. Liu, Lattice Boltzmann simulations of micron-scale drop impact on dry surfaces, J. Comput. Phys. 229 (20) (2010) 8045-8063.

[16] Y. Wang, C. Shu, H. Huang, C. Teo, Multiphase lattice Boltzmann flux solver for incompressible multiphase flows with large density ratio, Journal of Computational Physics 280 (2015) 404-423.

[17] X. Shan, H. Chen, Lattice Boltzmann model for simulating flows with multiple phases and components, Phys. Rev. E 47 (3) (1993) 1815-1819. 
[18] X. Shan, H. Chen, Simulation of nonideal gases and liquid-gas phase transitions by the lattice Boltzmann equation, Phys. Rev. E 49 (1994) 2941-2948.

[19] M. Sbragaglia, R. Benzi, L. Biferale, S. Succi, K. Sugiyama, F. Toschi, Generalized lattice Boltzmann method with multirange pseudopotential, Phys. Rev. E 75 (2007) 026702.

[20] X. He, S. Chen, R. Zhang, A lattice Boltzmann scheme for incompressible multiphase flow and its application in simulation of Rayleigh-Taylor instability, J. Comput. Phys. 152 (2) (1999) 642-663.

[21] A. Montessori, G. Falcucci, M. L. Rocca, S. Ansumali, S. Succi, Threedimensional lattice pseudo-potentials for multiphase flow simulations at high density ratios, Journal of Statistical Physics 161 (6) (2015) 1404-1419.

[22] G. Falcucci, S. Ubertini, S. Succi, Lattice Boltzmann simulations of phaseseparating flows at large density ratios: the case of doubly-attractive pseudo-potentials, Soft Matter 6 (2010) 4357-4365.

[23] R. Benzi, M. Sbragaglia, S. Succi, M. Bernaschi, S. Chibbaro, Mesoscopic lattice Boltzmann modeling of soft-glassy systems: Theory and simulations, J. Chem. Phys. 131 (2009) 104903.

[24] G. Falcucci, G. Bella, G. Shiatti, S. Chibbaro, M. Sbragaglia, S. Succi, Lattice Boltzmann models with mid-range interactions, Communications in Computational Physics 2 (2007) 1071-1084.

[25] C. E. Colosqui, G. Falcucci, S. Ubertini, S. Succi, Mesoscopic simulation of non-ideal fluids with self-tuning of the equation of state, Soft Matter 8 (2012) 3798-3809.

[26] R. R. Nourgaliev, T. N. Dinh, T. G. Theofanous, D. Joseph, The lattice Boltzmann equation method: Theoretical interpretation, numerics and implications, International Journal of Multiphase Flow 29 (1) (2003) 117-169. 
[27] H. Liu, Q. Kang, C. R. Leonardi, B. D. Jones, S. Schmieschek, A. Narváez, J. R. Williams, A. J. Valocchi, J. Harting, Multiphase lattice Boltzman$\mathrm{n}$ simulations for porous media applications, Computational Geosciences (2015) 1-29doi:10.1007/s10596-015-9542-3.

[28] K. N. Premnath, J. Abraham, Simulations of binary drop collisions with a multiple-relaxation-time lattice-Boltzmann model, Phys. Fluids 17 (12) (2005) 122105 .

[29] M. Pasandideh-Fard, Y. M. Qiao, S. Chandra, J. Mostaghimi, Capillary effects during droplet impact on a solid surface, Phys. Fluids 8 (1996) 650.

[30] J. Hua, B. Zhang, J. Lou, Numerical simulation of microdroplet formation in coflowing immiscible liquids, AIChE J. 53 (2007) 2534-2548.

[31] K. N. Premnath, J. Abraham, Lattice Boltzmann model for axisymmetric multiphase flows, Phys. Rev. E 71 (2005) 056706.

[32] S. Mukherjee, J. Abraham, Lattice boltzmann simulations of two-phase flow with high density ratio in axially symmetric geometry, Phys. Rev. E 75 (2007) 026701.

[33] T. Lee, C.-L. Lin, A stable discretization of the lattice Boltzmann equation for simulation of incompressible two-phase flows at high density ratio, J. Comput. Phys. 206 (1) (2005) $16-47$.

[34] J.-J. Huang, H. Huang, C. Shu, Y. T. Chew, S.-L. Wang, Hybrid multiplerelaxation-time lattice-boltzmann finite-difference method for axisymmetric multiphase flows, J. Phys. A-Math. Theor. 46 (2013) 055501.

[35] S. Srivastava, P. Perlekar, J. H. M. ten Thije Boonkkamp, N. Verma, F. Toschi, Axisymmetric multiphase lattice Boltzmann method, Phys. Rev. E 88 (2013) 013309.

[36] H. Liang, Z. H. Chai, B. C. Shi, Z. L. Guo, T. Zhang, Phase-field-based lattice Boltzmann model for axisymmetric multiphase flows, Phys. Rev. E 90 (2014) 063311. 
[37] H. Liang, B. C. Shi, Z. L. Guo, Z. H. Chai, Phase-field-based multiplerelaxation-time lattice Boltzmann model for incompressible multiphase flows, Phys. Rev. E 89 (2014) 053320.

[38] H. Liu, Y. Ju, N. Wang, G. Xi, Y. Zhang, Lattice Boltzmann modeling of contact angle and its hysteresis in two-phase flow with large viscosity difference, Phys. Rev. E 92 (2015) 033306.

[39] H. Liu, Y. Zhang, Droplet formation in microfluidic cross-junctions, Phys. Fluids 23 (8) (2011) 082101.

[40] A. Gupta, R. Kumar, Effect of geometry on droplet formation in the squeezing regime in a microfluidic T-junction, Microfluidics and Nanofluidics 8 (6) (2010) 799-812.

[41] H. Liu, A. J. Valocchi, C. Werth, Q. Kang, M. Oostrom, Pore-scale simulation of liquid CO2 displacement of water using a two-phase lattice Boltzmann model, Adv. Water Resour. 73 (2014) 144-158.

[42] Y. Ba, H. Liu, J. Sun, R. Zheng, Three dimensional simulations of droplet formation in symmetric and asymmetric T-junctions using the color-gradient lattice Boltzmann model, Int. J. Heat Mass Transf. 90 (2015) 931-947.

[43] H. Liu, Y. Zhang, A. J. Valocchi, Modeling and simulation of thermocapillary flows using lattice Boltzmann method, J. Comput. Phys. 231 (12) (2012) 4433-4453.

[44] H. Liu, Y. Zhang, Modeling thermocapillary migration of a microfluidic droplet on a solid surface, J. Comput. Phys. 280 (2015) 37-53.

[45] Y. Ba, H. Liu, J. Sun, R. Zheng, Color-gradient lattice Boltzmann model for simulating droplet motion with contact-angle hysteresis, Phys. Rev. E 8888 (2013) 043306. 
[46] J. U. Brackbill, D. B. Kothe, C. Zemach, A continuum method for modeling surface tension, J. Comput. Phys. 100 (2) (1992) 335-354.

[47] M. Latva-Kokko, D. H. Rothman, Diffusion properties of gradient-based lattice Boltzmann models of immiscible fluids, Phys. Rev. E 71 (2005) 056702 .

[48] C. M. Pooley, H. Kusumaatmaja, J. M. Yeomans, Contact line dynamics in binary lattice Boltzmann simulations, Phys. Rev. E 78 (5) (2008) 056709.

[49] M. L. Porter, E. T. Coon, Q. Kang, J. D. Moulton, J. W. Carey, Multicomponent interparticle-potential lattice Boltzmann model for fluids with large viscosity ratios, Phys. Rev. E 86 (2012) 036701.

[50] H. Liu, A. J. Valocchia, Y. Zhang, Q. Kang, Lattice Boltzmann phase-field modeling of thermocapillary flows in a confined microchannel, J. Comput. Phys. 256 (2014) 334-356.

[51] S. V. Lishchuk, C. M. Care, I. Halliday, Lattice Boltzmann algorithm for surface tension with greatly reduced microcurrents, Phys. Rev. E 67 (2003) 036701.

[52] I. Halliday, A. P. Hollis, C. M. Care, Lattice Boltzmann algorithm for continuum multicomponent flow, Phys. Rev. E 76 (2007) 026708.

[53] Q. Li, Y. L. He, G. H. Tang, W. Q. Tao, Improved axisymmetric lattice Boltzmann scheme, Phys. Rev. E 81 (2010) 056707.

[54] J. G. Zhou, Axisymmetric lattice Boltzmann method, Phys. Rev. E 78 (2008) 036701.

[55] Y. H. Qian, D. D'Humières, P. Lallemand, Lattice BGK models for NavierStokes equation, Europhys. Lett. 17 (1992) 479-484.

[56] X. F. Li, G. H. Tang, T. Y. Gao, W. Q. Tao, Simulation of Newtonian and non-Newtonian axisymmetric flow with an aisymmetric lattice Boltzmann model, Int. J. Mod. Phys. C 21 (10) (2010) 1237-1254. 
[57] J. G. Zhou, Axisymmetric lattice Boltzmann method revised, Phys. Rev. E 84 (2011) 036704.

[58] H. Huang, X.-Y. Lu, Theoretical and numerical study of axisymmetric lattice Boltzmann models, Phys. Rev. E 80 (2009) 016701.

[59] Y. Q. Zu, S. He, Phase-field-based lattice Boltzmann model for incompressible binary fluid systems with density and viscosity contrasts, Phys. Rev. E 87 (2013) 043301.

[60] L. Wu, M. Tsutahara, L. S. Kim, M. Ha, Three-dimensional lattice Boltzmann simulations of droplet formation in a cross-junction microchannel, Int. J. Multiphase Flow 34 (2008) 852-864.

[61] Z. Guo, C. Zheng, B. Shi, Discrete lattice effects on the forcing term in the lattice Boltzmann method, Phys. Rev. E 65 (2002) 046308.

[62] M. E. McCracken, J. Abraham, Multiple-relaxation-time lattice-Boltzmann model for multiphase flow, Phys. Rev. E 71 (2005) 036701.

[63] Z. Yu, L.-S. Fan, Multirelaxation-time interaction-potential-based lattice Boltzmann model for two-phase flow, Phys. Rev. E 82 (2010) 046708.

[64] P. Lallemand, L.-S. Luo, Theory of the lattice Boltzmann method: Dispersion, dissipation, isotropy, Galilean invariance, and stability, Phys. Rev. E 61 (6) (2000) 6546-6562.

[65] Z. Chai, T. S. Zhao, Effect of the forcing term in the multiple-relaxationtime lattice Boltzmann equation on the shear stress or the strain rate tensor, Phys. Rev. E 86 (2012) 016705.

[66] D. d'Humières, I. Ginzburg, M. Krafczyk, P. Lallemand, L.-S. Luo, Multiple-relaxation-time lattice Boltzmann models in three dimensions, Philos. Trans. R. Soc. A 360 (2002) 437-451. 
[67] A. J. C. Ladd, Numerical simulations of particulate suspensions via a discretized Boltzmann equation. (Part I \& II), J. Fluid Mech. 271 (1994) 285-339.

[68] A. Riaud, S. Zhao, K. Wang, Y. Cheng, G. Luo, Lattice-Boltzmann method for the simulation of multiphase mass transfer and reaction of dilute species, Phys. Rev. E 89 (2014) 053308.

[69] N. Ioannou, H. Liu, Y. Zhang, Droplet dynamics in confinement, Journal of Computational Science(In press). doi:10.1016/j.jocs.2016.03.009.

[70] K. Connington, T. Lee, A review of spurious currents in the lattice boltzmann method for multiphase flows, Journal of Mechanical Science and Technology 26 (12) (2012) 3857-3863.

[71] J. Yang, E. S. Boek, A comparison study of multi-component lattice Boltzmann models for flow in porous media applications, Computers \& Mathematics with Applications 65 (6) (2013) 882-890.

[72] C. A. Miller, L. E. Scriven, The oscillations of a fluid droplet immersed in another fluid, J. Fluid Mech. 32 (3) (1968) 417-435.

[73] I. Ginzburg, Equilibrium-type and link-type lattice Boltzmann models for generic advection and anisotropic-dispersion equation, Adv. Water Resour. 28 (2005) 1171-1195.

[74] C. Pan, L.-S. Luo, C. T. Miller, An evaluation of lattice Boltzmann schemes for porous medium flow simulation, Comput. Fluids 35 (2006) 898-909.

[75] A. Kuzmin, A. Mohamad, S. Succi, Multi-relaxation time lattice Boltzmann model for multiphase flows, Int. J. Mod. Phys. C 6 (2008) 875C902.

[76] A. Fakhari, T. Lee, Multiple-relaxation-time lattice Boltzmann method for immiscible fluids at high Reynolds numbers, Phys. Rev. E 87 (2013) 023304. 
[77] J. Latt, B. Chopard, Lattice Boltzmann method with regularized precollision distribution functions, Mathematics and Computers in Simulation 72 (2006) 165-168.

[78] H. Chen, R. Zhang, I. Staroselsky, M. Jhon, Recovery of full rotational invariance in lattice Boltzmann formulations for high Knudsen number flows, Physica A: Statistical Mechanics and its Applications 362 (1) (2006) $125-131$.

[79] R. Zhang, X. Shan, H. Chen, Efficient kinetic method for fluid simulation beyond the Navier-Stokes equation, Phys. Rev. E 74 (2006) 046703.

[80] H. Otomo, H. Fan, R. Hazlett, Y. Li, I. Staroselsky, R. Zhang, H. Chen, Simulation of residual oil displacement in a sinusoidal channel with the lattice Boltzmann method, Comptes Rendus Mécanique 343 (10-11) (2015) $559-570$.

[81] A. Montessori, G. Falcucci, P. Prestininzi, M. L. Rocca, S. Succi, Regularized lattice Bhatnagar-Gross-Krook model for two- and three-dimensional cavity flow simulations, Phys. Rev. E 89 (2014) 053317.

[82] A. Montessori, P. Prestininzi, M. L. Rocca, S. Succi, Lattice Boltzmann approach for complex nonequilibrium flows, Phys. Rev. E 92 (2015) 043308.

[83] J. Plateau, Experimental and theoretical statics of liquid fluids subject to molecular forces only, Vol. 1, Gauthier-Villars, Paris, 1873.

[84] L. Rayleigh, On the instability of jets, Proc. London Math. Soc. 10 (1878) $4-13$.

[85] N. Ashgriz, F. Mashayek, Temporal analysis of capillary jet breakup, J. Fluid Mech. 291 (1995) 163-190.

[86] M. Tjahjadi, H. A. Stone, J. M. Ottino, Satellite and subsatellite formation in capillary breakup, J. Fluid Mech. 243 (1992) 297-317. 
[87] P. Lafrance, Nonlinear breakup of a laminar liquid jet, Phys. Fluids 18 (1975) 428.

[88] D. F. Rutland, G. J. Jameson, Theoretical prediction of the sizes of drops formed in the breakup of capillary jets, Chem. Eng. Sci. 25 (11) (1970) 1689-1698.

[89] G. Falcucci, S. Ubertini, G. Bella, A. De Maio, S. Palpacelli, Lattice Boltzmann modeling of diesel spray formation and break-up, SAE Int. J. Fuels Lubr. 3 (1) (2010) 582-593. 